\title{
Building Nanostructures with Drugs
}

\section{Wang Ma, 1,\# Andrew G. Cheetham, ${ }^{2,3, \#}$ Honggang Cui ${ }^{*, 1,2,3,4,5}$}

1. Department of Oncology, The First Affiliated Hospital of Zhengzhou University, 1 Jianshe Eastern Road, Zhengzhou, Henan 450052, China

2. Department of Chemical and Biomolecular Engineering, The Johns Hopkins University, 3400 N Charles Street, Baltimore, MD 21218, USA

3. Institute for NanoBioTechnology, The Johns Hopkins University, 3400 N Charles Street, Baltimore, MD 21218, USA

4. Department of Oncology and Sidney Kimmel Comprehensive Cancer Center, Johns Hopkins University School of Medicine, Baltimore, Maryland 21205, USA

5. Center for Nanomedicine, The Wilmer Eye Institute, Johns Hopkins University School of Medicine, 400 North Broadway, Baltimore, Maryland 21231, USA

" Corresponding author email: hcui6@ jhu.edu; \# These authors contributed equally

\begin{abstract}
The convergence of nanoscience and drug delivery has prompted the formation of the field of nanomedicine, one that exploits the novel physicochemical and biological properties of nanostructures for improved medical treatments and reduced side effects. Until recently, this nanostructure-mediated strategy considered the drug to be solely a biologically active compound to be delivered, and its potential as a molecular building unit remained largely unexplored. A growing trend within nanomedicine has been the use of drug molecules to build well-defined nanostructures of various sizes and shapes. This strategy allows for the creation of self-delivering supramolecular nanomedicines containing a high and fixed drug content. Through rational design of the number and type of the drug incorporated, the resulting nanostructures can be tailored to assume various morphologies (e.g. nanospheres, rods, nanofibers, or nanotubes) for a particular mode of administration such as systemic, topical, and local delivery. This review covers the recent advances in this rapidly developing field, with the aim of providing an in-depth evaluation of the exciting opportunities that this new field could create to improve the current clinical practice of nanomedicine.
\end{abstract}

\section{Introduction}

The construction of complex supramolecular architectures through the self-assembly of basic molecular building blocks is a strategy that can be both practical and inspiring, affording nanostructures with properties that the individual unit does not necessarily possess [1]. Nature leads the way in harnessing the power of molecular assembly, as exemplified by the folding of polypeptide chains into functional proteins 
capable of specifically recognizing important biomolecules and mediating many biological events [2,3], forming the dynamic cytoskeletons from tubulins and actins essential for cellular and subcellular functions [4,5], and creating hair, nails, hooves and horns from the multilevel assembly of keratin proteins [6,7]. Inspired by these exquisite assembly systems, the accumulated knowledge from over fifty years of research has enabled the creation of a wide range of supramolecular structures from an ever expanding set of natural and synthetic building blocks, including small synthetic molecules [1], block copolymers [8-11], rationally designed peptides [12-18], carbohydrates [19], proteins [20] and DNA [21,22]. Concurrently, functional utilization of these supramolecular nanostructures has been extensively explored in diverse areas, particularly in the context of drug delivery [23-25]. The control over the size, morphology, surface chemistry, internal structures, and stability (including both disassembly kinetics and pathways) of supramolecular nanostructures bestows the potential to carry, protect and release a therapeutic cargo in a highly regulated manner [26-28]. This carrier-cargo relationship is one based on a separation of function, however, with the molecular building blocks purely responsible for delivery (assuming biocompatibility and inertness) and the drug for the ensuing bioactivity. Once its function has been fulfilled the carrier is considered waste material that needs to be cleared.

An emerging approach for the delivery of therapeutic agents has been to consider the drug itself as a key component of the self-assembly possessing the potential to create its own nanostructures. In this way, extraneous materials can be avoided without loss of function, perhaps leading to more effective therapies due to the increased drug loading, elimination of the leakage issue associated with encapsulated drugs, and the reduced need to remove synthetic carrier material. What is unclear is the extent to which the potential intermolecular interactions afforded by a drug's molecular structure can be harnessed to allow the rational design of a particular nanostructure and how general this approach can truly be given the wide range of structural types in clinical use. This review will discuss a number of strategies that have been adopted in which small molecule drugs have been utilized as building blocks in the construction of their own nanostructures, with an emphasis on those that involve true self-assembly pathways. In this context, molecular assembly is interpreted as the spontaneous and reversible association of molecules into aggregates (e.g. molecular clusters, discrete morphologies, or three dimensional networks) in order to minimize system free energy. It is important to note that nanostructures created through molecular selfassembly are distinct from those prepared by precipitation or emulsion methods in that the physiochemical properties of the former (e.g. size, shape, internal order, stability, aggregation number, surface chemistry) are reflective of the molecular characteristics of the building units and the assembly conditions. It is the interplay of the systemic entropy with the enthalpic interactions among the building units, water, and other molecules present in the assembly system that defines the eventual self-assembled morphology. We believe that the rapidly growing area of drug-promoted molecular assembly holds great 
promise for future research in nanomedicine and cancer chemotherapy, harnessing the intrinsic molecular interactions of a drug to build its own nanocarrier that, to paraphrase Lehn's definition of supramolecular chemistry [29], provides "functions beyond the drug".

\section{Nanostructure formed by free drugs}

Small molecule drugs exhibit a wide range of chemical structures that determine how their solubility, aggregation, and transportation behavior in solution ultimately determine their pharmacokinetic profiles after administration and pharmacodynamics in interfacing with their biological targets. Large hydrophobic moieties, such as hydrocarbons or polyaromatic segments that possess strong selfassociative interactions, can lead to solubility problems and a high propensity for self-aggregation in aqueous solution, whereas a number of charged groups, e.g. carboxylates or amines, bestow a greater solubility and reduce the potential for self-association. The study of the self-association behavior of drug molecules and potential candidates is an important subject in pharmaceutical research [30]. Knowledge of how these molecules interact with themselves will aid in improving their efficacy, in understanding their pharmacokinetic profile, and in developing the most suitable formulation for administration. For example, Shoichet and coworkers investigated the aggregation behavior of seven hydrophobic anticancer drugs and found that the formation of colloidal particles inhibited the in vitro cytotoxic potential [31]. These colloidal structures could be disrupted in the presence of surfactants, restoring the respective drug's toxicity. It was also found that the colloidal forms exhibited greater interactions with proteins, such as albumin, causing unpredictable pharmacokinetic behavior.

Drugs that are amphiphilic, containing well separated large hydrophobic and charged groups, could demonstrate reversible self-assembly behavior, resulting in formation of dynamic molecular clusters or even discrete supramolecular nanostructures of well-defined size and shape. For example, anthracyclines, a class of antibiotic-based antineoplastics characterized by a hydrophobic planar anthraquinone moiety and a hydrophilic amino-sugar ring (Fig. 1), possess decent aqueous solubility due to the ionizable amine group and are commonly observed to exist as dimers and higher oligomers in solution [32-35]. Studies suggest that these dimers exist in parallel and antiparallel orientations of the molecules [33,34]. It has been postulated that the structural differences in the packing among the various derivatives, e.g. daunomycin, Adriamycin (Doxorubicin), 4'-epiadriamycin, may account for the variation in pharmacological activity [34]. Hayakawa and coworkers have also reported the formation of doxorubicinbased hydrogels through the use of high $\mathrm{NaCl}$ concentrations [36], which induce further self-association via $\pi-\pi$ stacking of the aggregates. Similar dimerization behavior was also found for the anthracyclinealternative mitoxantrone [37], and for the water-soluble camptothecin derivative irinotecan (CPT-11) [38]. 
Folic acid, also known as vitamin $\mathrm{M}$ or $\mathrm{B}_{9}$, is another example that illustrates a drugs' self-assembling potential. The pterin ring is a critical feature of folic acid's self-assembly as its nitrogen and oxygen atoms are arranged in such a fashion as to allow the formation of a particular arrangement of hydrogen bonds, similar to that of nucleic acids in DNA. In alkaline solution, the self-association of folic acid proceeds via the formation of Hoogsteen-bonded tetrameric discs, which then stack through $\pi-\pi$ interactions and inter-disc hydrogen bonding to form chiral columns [39-42]. Further ordering results in liquid-crystalline mesophases, the nature of which can be influenced by folate concentration and the addition of salts.

Cui and coworkers recently found that the assembly of folic acid could be manipulated into forming nanofibers or lozenge-like platelets through the use of methanol/water mixtures [43]. In pure methanol, folic acid formed a self-supporting gel comprised of discrete nanofibers due to the formation of Hoogsteen intermolecular pairing of four folic acid molecules (Fig. 2A). Gradual addition of water to this gel led to the emergence of the lozenge-like platelets, which comprise closely packed short nanorods of tetramers. It was postulated that the increasing water content disrupted the long fibrous structures present to give the more compact platelet structures. Interestingly, similar treatment of the anticancer drug methotrexate did not induce formation of any well-defined nanostructures, likely due to the lack of hydrogen bond acceptors in methotrexate preventing formation of the tetrameric discs that are the basis of the higher order structures.

Dissolution of hydrophobic drugs in an organic solvent followed by dilution into water is a technique that can provide insight into a drug's self-aggregation behavior. For example, the quinoline alkaloid camptothecin (CPT), a topoisomerase I inhibitor, has been observed to self-associate through offset faceto-face $\pi-\pi$ interactions (J-type aggregation) [44]. More recently, Hao and coworkers characterized 100$400 \mathrm{~nm}$ wide helical nanoribbon structures formed from the injection of a concentrated DMSO solution of CPT into water (Fig. 2B) [45]. These nanostructures were composed of the active lactone form of CPT and exhibited reversible disassembly-assembly behavior in response to $\mathrm{pH}$ modulation. Increasing the $\mathrm{pH}$ led to the formation of the soluble, but inactive, carboxylate form of CPT; adding acid then reversed the equilibrium to reform the helical nanoribbons. Fourier-transform infra-red (FT-IR) spectroscopy indicated the presence of hydrogen bonding interactions, and X-ray diffraction experiments suggested that a lamellar structure existed within the nanoribbons. The authors thus proposed a packing model in which CPT molecules stack through $\pi-\pi$ interactions and interact with neighboring 'stacks' via hydrogen bonding.

Another interesting example was recently reported by Yan and colleagues in which two drugs, the water soluble CPT derivative irinotecan (Ir) and the water insoluble (hydrophobic) chlorambucil (Cb), were 
directly conjugated to one another to give an amphiphilic drug-drug conjugate (ADDC) (Fig. 2C) [46]. By diluting a DMSO solution of the ADDC with water, uniform nanoparticles with hydrodynamic diameters averaging $88 \mathrm{~nm}$ were obtained (Fig. 2D). Interestingly, concentrations below the critical aggregation concentration (CAC) were found to be inactive, whereas above the CAC they quickly became toxic, with some indication of synergistic action. It was thus proposed that only the nanoparticle form of the conjugate, rather than the free monomers, can be internalized by cells. This approach was also recently extended to create another ADDC from the anticancer drugs floxuridine (FdU) and bendamustine (BdM) [47].

In general, the self-assembly of free drug molecules is a somewhat haphazard process, with little control over the resultant morphology. Exploiting such behavior is therefore difficult at best and its study is accordingly rooted in understanding how the pharmacokinetic properties and treatment efficacy is affected. Should a discrete nanostructure even be formed, there is no guarantee that it would possess desirable physicochemical properties suitable for clinical treatments. To gain access to, and control over, more complex nanostructures it is therefore necessary to introduce modifications to the drug, in particular the design and synthesis of drug-conjugates where other elements are chemically grafted to the drug in order to imbue a greater propensity for controlled self-assembly and the formation of well-defined, homogeneous nanostructures. This approach aims to take advantage of the potential interactions that the drug has to offer, thus making the drug a vital structural element rather than just a functional unit. The following sections will focus on a number of recent strategies that are being used to further improve the delivery of drugs via construction of nanostructures, creating entities that have been termed onecomponent nanomedicines [48].

\section{Nanostructures Formed by Macromolecular Amphiphilic Prodrugs}

The advent of nanotechnological approaches to drug delivery began with the development of polymerdrug conjugates [49] in which hydrophobic anticancer drugs were covalently attached to water-soluble polymers, such as $N$-(2-hydroxypropyl)methacrylamide (HPMA) [50-52] and polyglutamic acid (PGA) $[53,54]$. In this strategy, the pharmacokinetic profile of the resultant conjugate (tissue accumulation, circulation half-life) is determined by the nature of the polymer, and controlled release can be achieved by choosing appropriate polymer-drug linkages. In theory, this combination of properties should lead to increased treatment efficacy and reduced side-effects, but issues with polydispersity in both the degree of polymerization and extent of drug attachment may cause significant batch-to-batch variability that could complicate the efficacy studies and thus hinder further optimization of the system. Accordingly, these have contributed to the protracted translation of polymer-drug conjugates into clinical use. 
In 1990, Yokoyama and Kataoka reported on the synthesis of a doxorubicin (DOX)-conjugated block copolymer that can assemble into micelles [55], one of the earliest examples of a rationally designed macromolecular amphiphilic prodrug (MAP). In this MAP, a poly(ethylene)glycol (PEG) was linked to poly(aspartic acid) (P(Asp)) to create the block copolymer, followed by the conjugation of DOX to the $\beta$ carboxylic acid function of the P(Asp) segment (Fig. 3A). In this particular system, the combination of the hydrophilic PEG block with hydrophobic DOX results in the formation of micelles with diameters averaging $50 \mathrm{~nm}$. These micelles were formed during the extensive aqueous dialysis of the $N, N$ dimethylformamide (DMF) reaction solution that followed DOX conjugation. Removal of DOX that was physically entrapped within the micelles initially proved difficult, though later optimization of the preparation was able to address this [56]. One issue that is commonly found for such systems is the difficulty to achieve complete conjugation of the drug to all available reactive sites due to steric hindrance. In one example of Kataoka's polymer-drug conjugate, there are approximately 17 aspartic acid residues available as conjugation sites yet only a maximum of $53 \%$ coverage with DOX could be obtained [56], though it should also be noted that this gives a respectable drug content of $39 \%(w / w)$. The unreacted, ionizable sites could potentially reduce the conjugate's capacity for self-assembly at lower concentrations. It was determined that a drug coverage of 40-50\% was necessary in order to overcome the electrostatic repulsions for formation of stable micelles [56]. Given the polydispersity native to such systems, some conjugates may not assemble at pharmaceutically relevant concentrations and potentially have very different physicochemical properties and circulation fates. Indeed, biodistribution studies showed that conjugates which could not maintain a micellar structure underwent rapid renal excretion, whereas the micellar form exhibited prolonged circulation times [57]. A later formulation of this MAP, known as NK911 [58], underwent Phase I clinical trials, exhibiting a higher and more prolonged plasma concentration than free DOX, though little difference in toxicity compared to free DOX was seen [59]. Comparisons with historical data from trials involving a liposomal DOX formulation (Doxil®) [60] showed that NK911 was cleared more rapidly at the same dosage, an observation attributed to its lower plasma stability. However, despite the lower plasma concentration, NK911 was able to more widely distribute DOX within tumorous tissues than Doxil, potentially due to greater leakage of NK911 from tumor vessels and its subsequent diffusion to distant cancer cells [61].

A number of similar MAPs to that developed by Yokohama and Kataoka have been reported in later studies. In particular, Matsumura and coworkers adapted this approach to incorporate SN-38, the active metabolite of irinotecan, though $\mathrm{P}(\mathrm{Asp})$ was replaced with poly-glutamate, $\mathrm{P}(\mathrm{Glu})$ [62]. This MAP exhibited significant advantages over free irinotecan in animal models with respect to both efficacy and toxicity. Phase I clinical trials generally showed milder side-effects in comparison to the free drug [63], 
and though Phase II trials are listed as having been completed within the last few years (ID\# NCT00951613 and NCT00951054), no data has yet been made available. More recently, Zhang and colleagues utilized this strategy to construct similar supramolecular nanostructures of the anticancer drugs doxorubicin [64] and camptothecin [65], employing reversible addition-fragmentation chain transfer (RAFT) polymerization to provide greater control over the polydispersity.

An interesting approach for the delivery of cisplatin (cis-diaminodichloroplatinum (II), CDDP) was also developed by Yokoyama and Kataoka, relying on the formation of coordination bonds between the Pt atom and the $\mathrm{P}(\mathrm{Glu})$ carboxylate side-chains (Fig. 3B) [66]. When mixed in water, CDDP cross-links the PEG-P(Glu) polymer chains, resulting in the spontaneous formation of micelles that were approximately $28 \mathrm{~nm}$ in diameter. When compared with those of the $50 \mathrm{~nm}$ micellar DOX-conjugates [55], it can be seen how the drug type can influence the nanostructure's properties. It was proposed, in this instance, that the ability of the Pt atom of CDDP to form two bonds to the polymer in addition to its smaller size allows the formation of a smaller, denser micelle. Release of CDDP was predicated on ligand exchange between the carboxylates and free chloride ions. Compared with an earlier version of this MAP based on PEG-P(Asp) [67-70], the release profiles indicated greater micellar stability, with Pt released in a sustained manner over $150 \mathrm{hr}$ (no burst release) and the nanostructures maintaining diameters over $25 \mathrm{~nm}$ for at least $50 \mathrm{hr}$. Consequently, this stability led to excellent in vivo efficacy, with further studies showing a reduction in both nephro- and neurotoxicity [71] and the potential for targeted delivery to lymph node metastases [72]. This promising platform, also known as NC-6004, is currently undergoing a number of clinical trials, both as a single treatment [73] and in combination with the antimetabolite gemcitabine, against a number of cancer types, including a Phase III trial against pancreatic cancer (ID\# NCT02043288).

The polymer-drug conjugates developed by Yokayama and Kataoka represent a very simple embodiment of the principles behind macromolecular amphiphilic prodrugs, relying on the enhanced permeation and retention (EPR) effect $[74,75]$ to passively target tumors. To imbue greater functionality to the assembled system, such as the capacity for active targeting of tumors, it is therefore necessary to explore other chemical entities and introduce greater complexity. One such example is provided by Liu and coworkers, who developed an amphiphilic polymer-drug conjugate based on CPT that possessed folic acid receptor targeting ligands, photo-triggered drug release and cross-linkers sensitive to both acidic and reductive environments (Fig. 4A) [76]. This strategy was based on co-assembly of two diblock copolymers, one that contained a folic acid (FA) ligand and one that contained a block of photo-caged CPT molecules. Cosolvation of these two entities in DMSO followed by the addition of aqueous buffer led to formation of micelles, which were then stabilized by crosslinking. These FA-decorated shell cross-linked micelles possessed a hydrodynamic diameter of $\sim 54 \mathrm{~nm}$, could be effectively internalized into A549 human lung 
adenocarcinoma epithelial cells, and demonstrated excellent in vitro cytotoxicity. While Liu's system is an impressive feat of design and synthesis, it also serves to illustrate that greater complexity can come at a cost, with an increased number of potential issues associated with the varying degrees of polydispersity (polymer molecular weight, number of conjugated drugs, crosslinking coverage) among the micelles that could complicate future translational studies. Despite such concerns, a tradeoff between greater functionality and increased complexity must be made to achieve the desired aims of a drug delivery platform.

The macromolecular components that constitute MAPs are not solely limited to conventional hydrophilic polymers. For instance, an interesting series of conjugates developed by Chilkoti and colleagues [77-79] exploits the thermally responsive properties of elastin-like peptides (ELPs) [80]. At low temperatures ELPs exist in a random conformation that renders them soluble, but at higher temperatures a conformational change occurs that lowers the solubility and can induce aggregation. In 2009, Chilkoti and coworkers reported an ELP-based drug-polypeptide conjugate that could self-assemble into spherical nanostructures [78]; a platform that was developed from previous work that utilized genetically engineered ELPs to thermally target solid tumors whose temperature had been raised by selective heat treatment $[77,81,82]$. The peptidic segment comprised the sequence $\operatorname{SKGPG}(\mathrm{XGVPG})_{160} \mathrm{WPC}\left(\mathrm{G}_{2} \mathrm{C}\right)_{7}$, with the cysteine residues included for the conjugation of up to eight drug molecules (Fig. 4B). A number of drug and drug-like molecules were tested, indicating that the octanol-water distribution coefficient, $\log$ $D$, is the critical determinant in the conjugate's ability to self-assemble $[78,79]$. The more hydrophobic the drug is, the better able it is to help induce nanostructure formation. In this example, the conjugation of DOX created a hydrophobic region that confers amphiphilicity to the conjugate. Upon dispersal in water, these conjugates self-assembled into nanoparticles with narrowly distributed hydrodynamic diameters of $21 \mathrm{~nm}$ and a CAC value of $3 \mu \mathrm{M}$ (Fig. 4C). In vivo experiments indicated that the nanoparticles experienced prolonged circulation, increased tumor accumulation and reduced uptake in non-tumor targets. Remarkably, a single-injection dose appeared to cure eight of nine mice for up to 66 days posttumor implantation, compared with median survival times of 21 and 27 days for the saline and free DOX control groups, respectively. Subsequent studies have further highlighted the promise this system possesses as a viable treatment option [83], especially with regards to the thermal targeting of tumors [84,85]. Heating of the tumor induces a phase transition in the conjugate, triggering aggregation that traps the nanoparticles within the tumor vasculature [85]. Upon cooling, a steep transvascular concentration gradient with the external soluble nanoparticles is created that facilitates further transport into the tumor environment (Fig. 4D-E). This behavior illustrates how further functionality can be effectively imparted 
through rational design choices, in particular the consolidation of multiple functions (hydrophilicity and targeting) into a single segment.

The nanostructure morphology formed through self-assembly of MAPs is another important feature that can have a significant effect on how it will perform. Since the self-assembly of block copolymers is typically under kinetic and thermodynamic control, it can be influenced by the various factors at play: cosolvent composition, polymer concentration, temperature and the rate of water addition [86]. Liu and coworkers exploited this behavior to induce a PEG-polyCPT diblock copolymer, synthesized via RAFT polymerization, to form nanospheres, nanodisks, staggered lamellae structures and large compound vesicles [87]. Liu found that the adopted structure could significantly affect the cellular internalization, not only in terms of the uptake efficiency, but also with regard to the mechanism of membrane transport. The most effective uptake was seen for the staggered lamellae structures, which effectively bypassed the endolysosomal compartments and dispersed well throughout cells. This greater uptake also resulted in this morphology exhibiting the greatest in vitro cytotoxicity. Whether this will translate into a greater in vivo efficacy will be interesting to learn, but for now Liu's polymer-drug conjugate provides a fascinating example of how processing conditions can be utilized to control the morphology, and how different structures could affect their cellular interactions and overall efficacy.

Macromolecular amphiphilic prodrugs have demonstrated great potential for delivering a drug payload through the drug-induced formation of nanostructures. Their development has benefitted greatly from the years of research that has been performed on polymeric delivery systems in general, with protocols for determining their properties already well established, and it is no surprise that a number are already in advanced clinical trials. For the most part, MAPs are synthetically facile to create, though can suffer from polydispersity issues. Advances in polymer synthesis techniques have alleviated this to some degree, providing greater control over the polymer size and enabling a more homogeneous polymer-drug conjugate to be produced, but the issue has not been completely eliminated and may still complicate translational studies. Stability during circulation is one concern that has already arisen, for instance, and others may arise from the reliance on the EPR effect as the dominant method of targeting. Further research into more active targeting strategies is desirable, though this could come at the cost of potentially increased synthetic complexity.

\section{Supramolecular Nanostructures Formed by Amphiphilic Prodrugs}

Amphiphilic prodrugs (APs) represent the next logical step in the evolution of self-delivering drugs; rather than conjugating the drug to a polymeric entity as in macromolecular amphiphilic prodrugs, a discrete entity that imbues the desired characteristics is utilized. This approach offers several advantages, one being that precise control over the drug content can be more easily achieved through rational 
molecular design. Furthermore, homogeneity is practically assured as the synthesized conjugate can be purified using HPLC and the nanostructure formed will contain the same drug content. There are no issues such as polydispersity in the macromolecular component or extent of drug conjugation per unimer. Given the lower molecular weight of APs, the drug becomes an integral part of the structure and as such can exert a great influence over the self-assembly pathway.

An early strategy in the development of APs was the lipidation of hydrophilic nucleoside-based drugs, an approach pioneered by both Jin and Couvreur. In all reports, nanoparticles were obtained using the precipitation method in which an organic solvent solution of the amphiphilic prodrug was added to aqueous buffer. These methodologies were applied to a number of different drug types, including antivirals [88,89], antineoplastics [90-94], antibiotics [95] and even anti-parasitics [96]. Later research efforts conjugated a hydrophilic element to hydrophobic drugs, again relying on the precipitation method to generate nanoscale objects [97-99]. Shen and coworkers, for example, created vesicle-like nanocapsules from oligoethylene glycol (OEG)-conjugated camptothecin [97]. These nanocapsules could also load a second anticancer drug, doxorubicin, for delivery in a fashion similar to that of the clinicallyrelevant liposomal formulation, Doxil [100]. It is evident, however, that the nanoprecipitation approach has limitations in effectively controlling the size, shape and surface chemistry of the resulting nanostructures due to the reliance of kinetic trapping in their assembly. By solely utilizing hydrophobic collapse as the primary driving force for forming nanostructures, there is less opportunity to direct how the rationally designed molecules arrange themselves within the resultant nanostructure. Consequently, the majority of ongoing research in amphiphilic prodrug design has focused on the introduction of additional elements that can contribute reversible and directional interactions to allow for a better controlled self-assembly pathway.

An early example of an amphiphilic prodrug that exhibited self-assembly behavior was reported by Cui and coworkers, who created a "drug amphiphile" platform in which a hydrophobic drug is conjugated to a short peptide with overall hydrophilicity and a preference for $\beta$-sheet formation [101]. The generic design of these drug amphiphiles is illustrated in Fig. 5A, and comprises the hydrophobic drug, a degradable linker for stimuli-triggered release, and a peptide segment that contains a structure-directing motif (e.g. $\beta$ sheet, $\alpha$-helix) and charged amino acids (or biologically relevant peptides). The peptide segment is a critical inclusion as the many natural and synthetic amino acids available allows for customization of the platform with regard to morphology, physicochemical properties and targeting ability. Cui's demonstration of this strategy conjugated the hydrophobic CPT to the $\beta$-sheet forming Tau peptide (VQIVYK) [102] via reducible disulfide linkages (Fig 5B) [101]. The drug content was precisely controlled through the introduction of branching points so that one, two or four CPT molecules were 
attached to give drug loadings of 23, 31 and 38\%, respectively. When dissolved in water, these conjugates assembled into one-dimensional (1D) filamentous structures in which the hydrophobic drug was sequestered within the core and thus protected from the external environment (Fig. 5C-E). Dilution studies of the assembled drug amphiphiles revealed they were stable at nano-molar concentrations, indicative of the strong associative interactions within the nanostructures. Interestingly, while the drug amphiphiles containing one and two CPT molecules gave nanofilaments (Fig. 5C and D), qCPT-buSSTau (four conjugated CPT molecules) was observed to adopt a nanotube morphology (Fig. 5E). The narrow diameter of these tubes (approx. $9.5 \pm 1 \mathrm{~nm}$ ) indicates that they are formed from a monolayer of amphiphiles, such that the internal channel is lined by hydrophobic CPT molecules. The origin of this change in morphology that the higher drug content induced was found to be due to the effect of $\pi-\pi$ interactions between the CPT molecules, presumably both intra- and intermolecular associations. Replacement of the Tau peptide with the more hydrophilic $\beta$-sheet-forming Sup35 peptide $\left(\mathrm{N}_{2} \mathrm{Q}_{2} \mathrm{NY}\right)$ [103], gave the same nanotube structure with the only difference being a longer contour length that may be a consequence of the greater solubility this peptide confers [43], suggesting that it is the arrangement of CPT molecules that is the major driving force behind this morphology.

The protection that the nanostructures provide the cleavable linker was illustrated by subjecting the conjugates to degradation by hydrolysis and reduction [101]. In all cases, glutathione-induced release was faster than hydrolysis alone as would be expected. It was seen that the more hydrophobic conjugates (dCPT-buSS-Tau and qCPT-buSS-Tau) provided the greatest protective effect, consistent with their more stable structures. A degradation study of mCPT-buSS-Tau at two different concentrations showed that the release was faster at low concentrations where the equilibrium between monomer and nanostructure would be in favor of a higher ratio of monomers to nanostructures. This clearly indicates how the drug amphiphiles can act as a reservoir for the bioactive drug, with the nanostructure undergoing dissociation to monomers at the target site that can then degrade to release free drug (Fig. 5F). However, an unintended consequence of the protection provided later became apparent, as a more mechanistic study into the release showed that the cytotoxicity of the mCPT-buSS-Tau conjugate was being compromised by the formation of hydrophobic CPT-containing disulfide species [104]. Upon reduction, the close proximity of molecules within the assembly promoted the formation of the symmetrical disulfide species, which would then remain closely associated with the nanostructure or form their own aggregates. Switching to an ethyl carbonate disulfide linker that can undergo rapid intramolecular degradation upon cleavage was found to restore the toxicity to almost that of the free CPT. These observations emphasize the importance of considering how the nanostructure will affect the desired release mechanism of the free drug. It is often assumed that the release mechanism will function as designed, but in a self-assembled 
system access to the linker may be limited by the density of packing, and with such high local monomer concentrations side reactions are also a possibility.

Replacement of the CPT in the drug amphiphile with the bulky taxane paclitaxel (PTX) was found to also give filamentous nanostructures with a fixed $41 \%$ drug loading [105]. However, while the morphology was unaltered there was a clear difference in the physical properties of the nanostructures, with the CAC value determined to be $10 \mu \mathrm{M}$, an almost 50-fold increase on that of the analogous CPT conjugate [101]. It is evident that the bulkier PTX molecule affects the internal packing order within the nanostructure and a higher concentration must therefore be achieved before the energetics become favorable enough to induce self-assembly. Furthermore, a drug amphiphile that contained two PTX molecules was only able to form spherical and very short filament-like structures upon dissolution in water [106]. No evidence of a $\beta$-sheet could be found, indicating that the two bulky PTX molecules prevented the peptide segment from forming the requisite hydrogen bonds that would promote elongation. These studies underscore how the design of drug amphiphiles must take into account the affect the drug will have on assembly. While it may be possible to switch out drugs of similar structure without creating problems, it must be assessed how drugs with dramatically different molecular structures will influence the assembly.

It is evident that the packing arrangement of molecules plays a critical role during the self-assembly process; behavior that can be exploited in order to produce new and interesting architectures $[8,18,107$ 109]. In one report, Cui and coworkers combined both CPT (flat aromatic with a preference for $\pi-\pi$ interactions) and PTX (bulky with the potential for hydrogen bonding) into a single drug amphiphile [106]. Borne out of the molecular frustration induced by having such disparate structural entities in close proximity, the conjugate initially forms small, flexible filamentous structures $7-8 \mathrm{~nm}$ in width (Fig. 6A), eventually evolving into long twisted two-filament fibrils $\sim 14 \mathrm{~nm}$ in width (Fig. 6B). It was postulated that the initial short filaments were the kinetic product of the hydrophobic collapse, with further rearrangement of the internal structure allowing the formation of $\beta$-sheet hydrogen bonds to give twisted two-filament fibrils as the thermodynamic product. A second approach was demonstrated by the catanionic mixing of oppositely charged derivatives of Cui's four-CPT bearing drug amphiphile, qCPTSup35-K $\mathbf{K}_{2}$ and $\mathbf{q C P T}-$ Sup35-E $\mathbf{2}_{2}$ [110], which led to the formation of large, multi-walled nanotubes (Fig. 6C); very distinct structures from the smaller nanotubes formed by the individual components. It was proposed that ion pair formation between the two oppositely charged drug amphiphiles leads to a reduction in the headgroup volume, thereby facilitating the formation of the bilayer(s) that constitutes the nanotube walls. 
The drug amphiphile that Cui is developing is designed to be a modular platform that can be adapted towards a particular mode of delivery. As it currently stands, the exclusive formation of filamentous structures is more suited toward local delivery applications given the potential that such structures have for entanglement and hydrogel formation [111-113]. However, there are reports of long nanofilaments being used for systemic delivery of cytotoxic agents [114,115], so with suitable modification of the surface chemistry applications requiring systemic delivery cannot be ruled out. Further fine tuning of the peptide sequence can also impart the ability to adopt different structures depending on the solution conditions. For example, Goldberger and coworkers [116] demonstrated that a peptide amphiphile could form either nanofibrous or spherical micellar structures depending on the solution $\mathrm{pH}$ and amphiphile concentration.

A number of other research groups have also investigated the use of drug-peptide amphiphiles that can self-assemble into well-defined nanostructures. A collaboration between the labs of Grinstaff and Parquette, for instance, led to the creation of a simple conjugate in which CPT was attached to the $\varepsilon$ amine of the $C$-terminal lysine in a Lys-Lys dipeptide (47\% drug loading) (Fig. 7A) [117]. Dissolution of the solid form in PBS and subsequent aging led to the formation of large nanotubule structures with diameters ranging from 80 to $120 \mathrm{~nm}$ and lengths of several micrometers. While they were found to have a very high CAC value $(350 \mu \mathrm{M})$, once formed they were exceptionally stable and would only dissociate in the low $\mathrm{nM}$ concentration range. In vitro assays confirmed that the nanotubes possessed good activity against a range of human colorectal and non-small cell lung cancer cell lines. Their large size and lack of flexibility, however, may prove to be detrimental for both systemic and local delivery applications. Dong and colleagues reported on the conjugation of hydroxyl-camptothecin (HCPT) to a multidomain peptide [118], the self-assembly of which results in short nanofilament structures that were 6-7 nm wide and no more than $50 \mathrm{~nm}$ long. The shorter nature of these nanostructures may hold advantages over longer nanofilaments for more effective cellular uptake. Ding and coworkers reported another example in which the conjugation of PTX to a tumor-homing cell-penetrating peptide (CPP) resulted in the formation of spherical nanostructures $\sim 120 \mathrm{~nm}$ in diameter [119].

An intriguing strategy has been developed by Zhang and coworkers, who exploited the properties of spherical nucleic acids (SNAs) [120,121] to create DNA-drug amphiphiles [122]. SNAs, which are normally based on a DNA shell around a solid core such as a gold nanoparticle, have been shown to have exceptional cellular uptake properties [123] and stability towards enzymatic degradation [124]. Zhang's approach was to replace the gold nanoparticle core with a drug reservoir. In this work, three molecules of CPT were conjugated to a strand of DNA via a photo-sensitive linkage (Fig. 7B), such that UV irradiation will trigger the irreversible release of free drug. The aqueous self-assembly behavior was found to be 
dependent upon the length of the DNA strand, with five base pairs giving a spherical micellar structure 28 $\pm 5 \mathrm{~nm}$ (determined by TEM) (Fig. 7C). Increasing the length of the strand eventually resulted in assembly being disfavored once the number of bases exceeded nine, which the authors attribute to reduced aggregation numbers as a consequence of the increasing volume within the head of the amphiphile. In the presence of $\mathrm{Mg}^{2+}$ ions, however, self-assembly could be observed in all the DNA-drug amphiphiles synthesized (up to 20 bases). This was attributed to the ability of the divalent cation to bridge neighboring strands and overcome the entropic penalty incurred during the assembly of densely packed nanostructures. Surprisingly, the largest DNA-drug amphiphile, DNA $\mathbf{2 0}_{\mathbf{2}}-\mathbf{C P T}$, adopted a rod-like morphology with diameters of $8 \pm 1 \mathrm{~nm}$ and lengths $53 \pm 14 \mathrm{~nm}$ (Fig. 7D), an observation that is still under investigation. In vitro studies of the $\mathbf{D N A}_{20}-\mathbf{C P T}$ showed that they retained their capacity for highly efficient cellular uptake, exhibiting comparable activity to free CPT against Sk-Br3 ovarian cancer cells under UV irradiation. Zhang's focus on the activity of the longer DNA-drug conjugate lies in the utility of DNA sequences with lengths of 18-25 bases for gene regulation applications, suggesting potential use of the DNA-drug amphiphiles for combination therapy-simultaneous chemo- and genetherapies in this instance. Overall, DNA-drug amphiphiles possess great potential as drug delivery vehicles, though a major hurdle is the promiscuity that SNAs display towards cellular internalization [125,126]. More selective delivery has been demonstrated using conjugated antibodies [127], an encouraging result for the development of a viable delivery platform.

Amphiphilic prodrugs represent an intriguing new class of drug delivery vehicles, one that holds the promise of customization to suit a particular therapeutic application. The modular design that many of the platforms in development exhibit imbues them with the capacity for fine tuning of the nanostructure morphology and physicochemical properties, making them adaptable for local and systemic delivery. Given that APs are still at an early stage of development, there are a number of concerns that have been raised, particularly with regard to the stability of the resulting nanostructures. While the CAC of the assembly is typically seen as the defining property, it is perhaps the resistance to disassembly upon dilution that is of greater import. Asymmetry in these two phenomena is quite possible for instance [117], as though a relatively high concentration may be required for assembly to occur, the strong intermolecular interactions that may form during this process could result in a much lower disassembly concentration. How this will play into in vivo efficacy remains to be determined, however, and may be challenging to ascertain. Concurrent advances in nanotechnological approaches to in vivo imaging may provide some assistance with this $[11,128]$, as similar concerns will exist over the circulation fate of nanoprobes that possess similar physicochemical properties to nanostructures formed by APs. 
One other aspect that needs to be addressed is the adaptability of the platform with respect to the incorporated drug. The smaller nature of APs means that the drug can play a much larger role in the selfassembly process when compared with MAPS, and while the polymeric nature of the latter may compensate for any reluctance toward assembly from the drug, this will not be the case in the former. Thus far, the majority of the work reported has focused on the use of the hydrophobic CPT as a model drug owing to its planar nature, its preference for $\pi-\pi$ interactions, and the ease with which it can modified. This does, however, limit our knowledge of how the drug can influence the overall nanostructure and its physicochemical properties. Cui's work has shown that an increased drug loading can affect the self-assembled morphology as the drug's own interactions become more dominant [101], and that steric effects too can cause variations in the self-assembly pathway [106]. More investigation is therefore required, as it will only be once a wider range of drug structure classes have been explored that we will learn if amphiphilic prodrug platforms have the expected broad utility, or if they will be limited to certain drug types.

\section{Supramolecular Hydrogels formed by Amphiphilic Prodrugs}

Molecular hydrogelators are a special class of amphiphilic molecules that are designed to spontaneously form a self-supporting gel when dispersed in water. This typically occurs through self-assembly into filamentous nanostructures that then entangle to create a three-dimensional network that traps the water and prevents its free movement. Compared with their polymer-based counterparts, molecular hydrogelators offer several advantages, such as an inherent biodegradability, reversible formationdissociation, and lower immunogenicity. In a little over two decades, development of these materials has led to numerous medically-relevant applications, including tissue engineering scaffolds [129-131], matrices for biomineralization [132,133] and wound healing [134], and as local drug delivery vehicles $[135,136]$. It is their ability to entrap and release biomolecules and small molecules, in combination with their three-dimensional network structure, which allows their use for biomedical applications. The utility of this approach towards drug delivery, however, is limited owing to the physical method of entrapment that, like polymeric delivery vehicles, can suffer from low loading and poor control over the release characteristics. Additionally, the hydrophilic environment of the gel can result in the crystallization of drugs with low water solubility, which can have a detrimental effect on the therapeutic effectiveness. For instance, the hydrophobic PTX has been observed to form crystals within hydrogels, much like those formed in aqueous solution [137], and it was speculated that this may be the reason why in some studies hydrogels with a lower drug content exhibited greater activity than those with a higher loading [138].

Modifying drug molecules themselves to bestow the capacity for hydrogelation provides a means for circumventing both the drug crystallization and low loading issues associated with excipient hydrogels 
[12]. Drug-based molecular hydrogelators have been in development for almost twenty years now and, in contrast to non-gelating amphiphilic prodrugs, have encompassed a broad range of drug molecules, including inhibitory hormones [139,140], antibiotics [141-143], anti-neoplastics [118,144-147], antiinflammatories [148-151], and anti-virals [152]. The majority of these hydrogelators are peptide-based, as their propensity for hydrogen bonding is ideal for the formation of the filamentous structures generally required for gelation to occur. Indeed, a number of peptide drugs are known to form hydrogels themselves (autogels) in aqueous conditions, such as lanreotide (Fig. 8A) [139], ganirelix (Fig. 8A) [140], and degarelix [140], with lanreotide being approved by the FDA for the treatment of acromelagy [153] and neuroendocrine tumors [154] as an injectable sustained release hydrogel (Somatuline $®$ Depot, Ipsen). A common feature between these three examples is a naphthalene moiety that, for lanreotide at least, has been shown to significantly contribute to the self-assembly through $\pi-\pi$ interactions [155]. Xu and coworkers demonstrated that the attachment of a pyrene group to the glycopeptide antibiotic vancomycin confers an ability to form hydrogels (Fig. 8B) [141]. Surprisingly, this modified-vancomycin was found to have nearly thousand-fold better activity against vancomycin-resistant enterococci bacteria than vancomycin alone; an observation later postulated as arising from self-assembly of the conjugate on the bacterial cell surface [142].

The modification of non-peptidic drugs to bestow gelation potential typically involves the conjugation of a short peptide sequence capable of forming strong, directional intermolecular interactions. A common motif is the dipeptide Phe-Phe, often in combination with a planar aromatic group at the $N$-terminal for additional hydrophobicity [130,131,156]. This dipeptide is the key recognition motif of $A \beta$ amyloid [157] and has been observed to form one dimensional nanostructures through the combination of hydrogen bonding and $\pi-\pi$ interactions [158,159]. Xu and colleagues utilized this motif to create the PTXcontaining hydrogelator NapFFK(succ-PTX)pY (Fig. 8C) [144], conjugating the succinylated-drug to the peptide through the $\varepsilon$-amine of lysine. Additionally, the capacity for triggered gelation was introduced through incorporation of a phosphorylated tyrosine residue $(p \mathrm{Y})$ that provides hydrophilicity, but can be cleaved by a phosphatase enzyme to reduce the solubility and induce self-assembly. Spectroscopic studies showed that the resulting filamentous structures possessed anti-parallel $\beta$-sheets, indicating that the PTX is likely displayed at the surface of the nanostructures. While this may suggest it would be readily accessible, entanglement between filaments will be favored in order to reduce hydrophobic-hydrophilic contacts between the water and PTX, helping to promote gel formation. This Nap-FFY platform or similar have been further exploited by $\mathrm{Xu}$ and others in the field to create hydrogelators based on a number of drugs, including the anti-inflammatory olsalazine [148], the antibiotic kanamycin [143], and the anticancer drugs bortezemib [146] and cisplatin [145]. 
An alternative conjugation approach is to attach the drug to the $N$-terminal of the peptide, rather than to one of the amino acid side chains. This does, however, place certain limitations on the drug structure in order to maintain the self-assembly properties of the conjugate, requiring flat hydrophobic drugs. One class of drugs that seem particularly suited for this are non-steroidal anti-inflammatory drugs (NSAIDs), which tend to be rather simple aromatic molecules. $\mathrm{Xu}$ and colleagues conjugated the NSAIDs naproxen (Npx), flurbiprofen, ibuprofen and salicylic acid (as an aspirin mimic) to the $N$-termini of the FF peptide [151]. Examination of the mechanical properties revealed that the drug's ability to contribute to the $\pi-\pi$ interactions during self-assembly greatly affect the rheological parameters of the resulting hydrogels, with the naphthyl-based naproxen conjugate furnishing the strongest gel and the phenyl-based ibuprofen the weakest. Yang, too, has reported hydrogelators in which the drug is attached to the $N$-terminal of the peptide, using the anticancer drugs curcumin [160] and gemcitabine [161], both of which possess very different molecular structures to the NSAIDs, affording gels with G' and G' values two orders of magnitude lower than those of Xu's naproxen conjugate. The differing peptide sequences make a direct comparison difficult, however, though both curcumin and gemcitabine possess smaller aromatic systems than naproxen and so this may a contributing factor.

Platforms for potential combination therapy applications have also emerged with the creation of molecular hydrogelators that contain two complementary drugs. For instance, Yang and coworkers synthesized a conjugate that possessed dexamethasone and either of PTX or HCPT (Fig. 8D) [147]. Dexamethasone is a steroidal drug with anti-inflammatory and immunosuppressant properties that is often used in combination with other anticancer drugs to reduce the associated side-effects [162]. While no benefit was seen to its inclusion in terms of in vitro toxicity, it would be anticipated that there may be advantages in vivo with reduced side effects. More recently, $\mathrm{Xu}$ and colleagues conjugated the anti-HIV drug, lamivudine (3TC), to the lysine $\varepsilon$-amine of their Npx-ffkpy hydrogelators [149] to create a hydrogel that can deliver both an anti-inflammatory and anti-HIV drug [152]. The intent here being to create a multifunctional delivery system to address several issues associated with the use of vaginally-applied anti-HIV gels as prophylactics. Cleavage of the phosphate group by the prostatic acid phosphatase (PAP) enzyme present in seminal fluid results in the strengthening of the weak gel formed by Npx-ffk(3TC)py, thereby minimizing its dilution. Release of the $\mathrm{Npx}$ would reduce local inflammation that would otherwise increase the chances of the HIV virus encountering and entering T cells, and the 3TC would act against the virus itself. This concept of incorporating two complementary drugs offers an intriguing path for future hydrogel platforms that could provide synergistic effects during treatment.

Despite the proliferation of hydrogels that are based on the diphenylalanine motif, there are a number of other strategies that have generated useful platforms for drug delivery. One such simple approach is the 
succinylation of a hydrophobic drug in order to confer amphiphilicity. Chen and coworkers treated the corticosteroid trimamcinolone acetonide (TA) with succinic anhydride to form a progelator that, upon hydrolysis of the succinate ester bond, would form a hydrogel [150]. This gel was used to treat uveitis in a rat model without exhibiting any of the associated complications that usually arise from TA treatment. Yang and colleagues also found that succinylated PTX can be induced to form a gel through sonication of a suspension [163]. The gel formed, however, was very weak and required the addition of a polymer additive (hyaluronic acid, HA) to strengthen it. Surprisingly, this had the added bonus of improving the anti-tumor activity for gels with $30 \%$ or higher HA content, possibly due to the ability of HA to target the CD44 antigen that is often over-expressed in cancer cells [164]. In another example of simple conjugation to a drug molecule, Yang and coworkers connected the oxidized form of glutathione to PTX via a succinyl linkage that upon reduction could form a hydrogel [165]. The mechanical properties of this hydrogel were such that it was suitable for administration as an injectable; being able to flow as a liquid under shear stress but recovering within a few minutes once the stress was removed (shear thinning behavior). Intra-tumoral injection of the gel in a mouse model was found to significantly inhibit tumor growth and metastasis, with noticeably lessened or absent side effects.

Stupp and coworkers have demonstrated that their hydrogel-forming peptide amphiphile platform can be adapted to sustainably release a number of drug types [166-169]. Stupp's peptide amphiphiles are typically $\beta$-sheet forming peptides with charged amino acids at the $C$-terminal end and a palmitoylated $\left(\mathrm{C}_{16}\right) \mathrm{N}$-terminus that self-assemble into core-shell nanofiber structures. Conjugation of the drugs was achieved through the lysine $\varepsilon$-amine of $\mathrm{C}_{16}-\mathrm{V}_{\mathrm{x}} \mathrm{A}_{\mathrm{x}} \mathrm{E}_{\mathrm{x}} \mathrm{K}(\mathrm{x}=2$ or 3$)$, resulting in nanostructures in which the drug is exposed at the periphery and thus accessible to the agents required for cleavage. Gelation was achieved by the addition of $\mathrm{CaCl}_{2}$ to shield the negative charges of the glutamates and reduce inter-fiber repulsions. This method was utilized for the sustained release of the NSAIDs nabumetone [167] and naproxen [168], the steroidal anti-inflammatory dexamethasone [166], and therapeutic carbon monoxide [169]. It should also be noted that Cui's drug amphiphile platform [101] can be similarly adapted for hydrogel-mediated delivery of drugs, with the filamentous nanostructures formed capable of entangling under appropriate conditions in the same manner as Stupp's peptide amphiphiles.

An interesting example worth highlighting is that of a hydrogel formed through the gelation of nanospheres rather than nanofilaments [170], a somewhat intriguing exception to the norm. Created by Yang and coworkers, the conjugate FA-GpYK(succ-PTX), where FA is folic acid, was observed to form a clear gel upon phosphatase-catalyzed removal of the tyrosine phosphate group (Fig. 9A). AFM imaging revealed the presence of highly uniform nanospheres with diameters of $50 \mathrm{~nm}$ (Fig. 9B), a structure that may arise from the capability of folic acid to form tetrameric structures through Hoogsteen hydrogen 
bonding, with further interactions giving a dendrimer-like structure (Fig. 9C). Rheological studies indicated that the gel was very weak, no doubt due to the inability of nanoparticles to form an entangled matrix like those of nanofilament-based hydrogels.

There have been a number of reports that feature multicomponent hydrogels, formed through the interaction of two or more species. When Ding and coworkers attempted to use the peptide NapGFFYGRGD to encapsulate the anticancer drug DOX, they were surprised to find that it could form a hydrogel [171]. The peptide alone forms nanofilaments but is unsuitable for gel formation due to poor inter-fiber interactions, though has shown utility for the encapsulation of hydrophobic drugs [172]. Instead of drug encapsulation, however, they found that DOX formed large nanospheres $(\sim 150 \mathrm{~nm}$ in diameter) on the fiber surface, essentially acting as cross-linkers between neighboring fibers and thus enabling gel formation (Fig. 9D). Drug release was observed to be faster from weaker gels; thereby correlating with the DOX concentration of the hydrogels, with higher concentrations giving stronger gels. Another example is provided by the Shi Lab, who adopted a highly supramolecular approach (Fig. 9E) [173]. Conjugating a low molecular weight PEG chain to CPT, they then mixed a solution of this micelleforming conjugate with $\alpha$-cyclodextrin $(\alpha$-CD), a cyclized glucose hexamer with a central hydrophobic cavity. Multiple $\alpha$-CD molecules thread onto the extended PEG chains to form what is known as a polypseudorotaxane, with interactions between $\alpha$-CD units then acting to cross-link the micelles and form a hydrogel matrix. While not a strong gel, it did exhibit shear thinning behavior that would make it suitable for injectable administration. Furthermore, this gel could encapsulate the water soluble anticancer drug, 5-fluorouracil (5-FU), which is often used in combination with CPT [174,175], with no disruptive effect on the hydrogel. This three-component system displayed superior in vitro anticancer activity when compared to each drug alone, suggesting a synergetic effect of the two drugs, though only at low 5-FU concentrations as higher concentrations resulted in an antagonistic effect that is known for this drug combination [175]. In a final example, Jana and Dastidar developed a xerogel platform in which naproxen and its $\beta$-alanine derivatives were induced to form gels by the addition of the biocompatible amine, serinol [176]. The resulting salt could form an extended hydrogen bonding network, giving nanofibers that could entangle to form gels with weak to moderate mechanical properties. A topical gel formulation was prepared and used to successfully treat chemically-induced psoriasis-like inflammation in mice. Gel treated mice completely recovered within 14 days, including hair regrowth, while those in the control group died after 2 days.

Supramolecular hydrogels formed by amphiphilic prodrugs are well positioned to become a successful nanomedicine, particularly given the wide usage of conventional hydrogels in clinical treatments. This platform can potentially be applied to many disease areas due to the broad range of drug classes that can 
be converted into hydrogelating materials. Before this can come to fruition, however, more in vivo studies are required to ascertain just how these supramolecular materials will function in a physiological environment. The mechanical properties of these materials are important in this context as shear stresses both during and after administration may affect the integrity of the gel and lead to a faster rate of drug release or even gel clearance. Release of the drug is another critical function of hydrogels, if the gel is too stable then the release may be slower than desired and result in poor efficacy. If it is to be triggered by a particular chemical or biological entity rather than simple hydrolysis, then the gel must be sufficiently permeable to that stimulus. A balance is therefore necessary with regard to the mechanical properties, and it is clear from the work reported that the drug can have a strong influence on the gel strength. Accordingly, strategies must be devised to address any issues that may arise for a particular drug class in order to develop the most effective therapeutic hydrogel possible.

\section{Future outlook}

Utilizing drugs as building units for the construction of nanostructures represents a paradigm shift in the growing field of nanomedicine, one in which drugs are no longer considered as passive cargos but as active participants in their own delivery to therapeutic targets. By exploiting the molecular properties that drug molecules possess in the context of self-assembly and combining them with complementary entities, it is now possible to construct discrete nanostructures that possess a high and precisely defined drug content through rational molecular design. Three main strategies have emerged to accomplish this, each having their own unique advantages and disadvantages. For instance, while macromolecular amphiphilic prodrugs are the most well-developed of the three due to their similarity with other polymer-based delivery systems, the prevalence of spherical nanostructures may limit their use and optimization to address the sophisticated challenges associated with systemic delivery applications. Conversely, supramolecular hydrogels formed by amphiphilic prodrugs are ideally suited to local delivery for treating cancers or other diseases that can benefit from topical or local administration of therapeutics. Amphiphilic prodrugs straddle the line between MAPs and hydrogelators, with their tunable morphologies enabling the adaptation for either route of administration. However, it has yet to be established how adaptable this platform is due to the strong influence of the drug molecule as a structural element and the limited number of drug structures tested so far. Given the push towards personalized medicine that is currently underway, it is important that the principles underlying how a drug molecule can affect the assembly of a nanostructure are determined and understood. The drug delivery community as a whole would benefit greatly from the availability of generic platforms that can deliver a particular drug type. After all, even though medicines may become personalized, they will still need to be effectively delivered to target sites so as to exert the desired biological effects. 
For all systems, a big question to be answered is how will the physiological environment impact the physicochemical properties and eventually the performance of these drug-containing nanostructures? How is the integrity of the nanostructures affected by interactions with proteins and the body's natural defense systems? Part of this lies with the critical aggregation concentration of the self-assembled nanostructure, for which a careful balance is required as too low a value may impede the drug release, while too high will lead to rapid dissociation. The latter is clearly undesirable for the purpose of systemic delivery, but could be an advantageous property for local delivery, particularly if the dissociation kinetics can be used to tune the drug release rate. For systemic delivery, if the goal is to reduce the CAC as much as possible, then strategies to facilitate dissociation at the desired location must also be considered in parallel. One potential method is the inclusion of self-immolating components that will breakdown in specific environments, thereby negating the need for equilibrium driven dissociation.

While many challenges are still ahead, the direct use of drugs to build well-defined nanostructures presents a significant extension from both fields of molecular assembly and medicine. Aside from a few examples formed from macromolecular amphiphilic prodrugs that have been evaluated in clinical trials, those created by assembly of small molecular amphiphilic prodrugs are still focused on fundamental studies of their assembly behavior and in vitro evaluation of their potency against cancer cell lines. Clearly, the next logical step will be testing these supramolecular materials in animals to collect information on their circulation fate and therapeutic efficacy, which will prove essential for guiding the design of next generation of drug-containing nanostructures for improved performance.

\section{Acknowledgement}

We acknowledge financial support from the National Science Foundation (DMR 1255281) and the National Institutes of Health (NIH/1R21CA191740).

\section{References}

[1] T. Aida, E.W. Meijer, S.I. Stupp, Science 335, (2012), 813-817.

[2] B. Alberts, A. Johnson, J. Lewis, M. Raff, K. Roberts, P. Walter. in Molecular Biology of the Cell, 4th edition Ch. 3, (Garland Science, 2002).

[3] F.U. Hartl, A. Bracher, M. Hayer-Hartl, Nature 475, (2011), 324-332.

[4] R.C. Weisenberg, Science 177, (1972), 1104-1105.

[5] S. Sahu, S. Ghosh, D. Fujita, A. Bandyopadhyay, Sci. Rep. 4, (2014).

[6] R.D. Bruce Fraser, D.A.D. Parry, J. Struct. Biol. 142, (2003), 319-325.

[7] L. Tombolato, E.E. Novitskaya, P.-Y. Chen, F.A. Sheppard, J. McKittrick, Acta Biomater. 6, (2010), 319-330.

[8] F.S. Bates, M.A. Hillmyer, T.P. Lodge, C.M. Bates, K.T. Delaney, G.H. Fredrickson, Science 336, (2012), 434-440. 
[9] H. Cui, Z. Chen, S. Zhong, K.L. Wooley, D.J. Pochan, Science 317, (2007), 647-650.

[10] T. Gadt, N.S. Ieong, G. Cambridge, M.A. Winnik, I. Manners, Nat. Mater. 8, (2009), 144-150.

[11] S.M. Janib, S. Liu, R. Park, M.K. Pastuszka, P. Shi, A.S. Moses, M.M. Orosco, Y.A. Lin, H. Cui, P.S. Conti, Z. Li, J.A. MacKay, Integrative Biology 5, (2013), 183-194.

[12] F. Zhao, M.L. Ma, B. Xu, Chem. Soc. Rev. 38, (2009), 883-891.

[13] B.E.I. Ramakers, J.C.M. van Hest, D.W.P.M. Lowik, Chem. Soc. Rev. 43, (2014), 2743-2756.

[14] A. Trent, R. Marullo, B. Lin, M. Black, M. Tirrell, Soft Matter 7, (2011), 9572-9582.

[15] V.A. Kumar, N.L. Taylor, S. Shi, B.K. Wang, A.A. Jalan, M.K. Kang, N.C. Wickremasinghe, J.D. Hartgerink, ACS Nano 9, (2015), 860-868.

[16] H. Cui, M.J. Webber, S.I. Stupp, Pept. Sci. 94, (2010), 1-18.

[17] T.J. Moyer, H. Cui, S.I. Stupp, The Journal of Physical Chemistry B 117, (2013), 4604-4610.

[18] H. Cui, A.G. Cheetham, E.T. Pashuck, S.I. Stupp, J. Am. Chem. Soc. 136, (2014), 12461-12468.

[19] R.A. Pires, Y.M. Abul-Haija, D.S. Costa, R. Novoa-Carballal, R.L. Reis, R.V. Ulijn, I. Pashkuleva, J. Am. Chem. Soc. 137, (2015), 576-579.

[20] J.W. Kelly, Curr. Opin. Struct. Biol. 8, (1998), 101-106.

[21] P.W.K. Rothemund, Nature 440, (2006), 297-302.

[22] T.R. Pearce, E. Kokkoli, Soft Matter 11, (2015), 109-117.

[23] D. Peer, J.M. Karp, S. Hong, O.C. Farokhzad, R. Margalit, R. Langer, Nat. Nanotechnol. 2, (2007), 751-760.

[24] J.W. de Vries, F. Zhang, A. Herrmann, J. Control. Release 172, (2013), 467-483.

[25] R. Lin, H. Cui, Curr. Opin. Chem. Eng. 7, (2015), 75-83.

[26] Y. Bae, S. Fukushima, A. Harada, K. Kataoka, Angew. Chem. Int. Ed. 42, (2003), 4640-4643.

[27] R. Haag, Angew. Chem. Int. Ed. 43, (2004), 278-282.

[28] C.-M.J. Hu, R.H. Fang, B.T. Luk, L. Zhang, Nat. Nanotechnol. 8, (2013), 933-938.

[29] J.-M. Lehn, Angew. Chem. Int. Ed. 27, (1988), 89-112.

[30] S. Schreier, S.V.P. Malheiros, E. de Paula, Biochimica et Biophysica Acta (BBA) - Biomembranes 1508, (2000), 210-234.

[31] S.C. Owen, A.K. Doak, P. Wassam, M.S. Shoichet, B.K. Shoichet, ACS Chem. Biol. 7, (2012), 1429-1435.

[32] M. Menozzi, L. Valentini, E. Vannini, F. Arcamone, J. Pharm. Sci. 73, (1984), 766-770.

[33] M.P. Evstigneev, V.V. Khomich, D.B. Davies, Russ. J. Phys. Chem, 80, (2006), 741-746.

[34] P. Agrawal, S.K. Barthwal, R. Barthwal, Eur. J. Med. Chem. 44, (2009), 1437-1451.

[35] Z. Fulop, R. Gref, T. Loftsson, Intl. J. Pharm. 454, (2013), 559-561.

[36] E. Hayakawa, K. Furuya, T. Kuroda, M. Moriyama, A. Kondo, Chem. Pharm. Bull. 39, (1991), 1282-1286.

[37] M. Enache, E. Volanschi, Rev. Roum. Chim. 55, (2010), 255-262.

[38] R. Aiyama, H. Nagai, S. Sawada, T. Yokokura, H. Itokawa, M. Nakanishi, Chem. Pharm. Bull. 40, (1992), 2810-2813.

[39] F. Ciuchi, G. Di Nicola, H. Franz, G. Gottarelli, P. Mariani, M.G. Ponzi Bossi, G.P. Spada, J. Am. Chem. Soc. 116, (1994), 7064-7071.

[40] S. Bonazzi, M.M. DeMorais, G. Gottarelli, P. Mariani, G.P. Spada, Angew. Chem. Int. Ed. 32, (1993), 248-250.

[41] G. Gottarelli, E. Mezzina, G.P. Spada, F. Carsughi, G.D. Nicola, P. Mariani, A. Sabatucci, S. Bonazzi, Helv. Chim. Acta 79, (1996), 220-234.

[42] Y. Kamikawa, M. Nishii, T. Kato, Chem.-Eur. J. 10, (2004), 5942-5951.

[43] L.L. Lock, M. LaComb, K. Schwarz, A.G. Cheetham, Y.-a. Lin, P. Zhang, H. Cui, Faraday Discuss. 166, (2013), 285-301.

[44] I. Nabiev, F. Fleury, I. Kudelina, Y. Pommier, F. Charton, J.F. Riou, A.J.P. Alix, M. Manfait, Biochem. Pharmacol. 55, (1998), 1163-1174.

[45] M.F. Ma, P.Y. Xing, S.G. Xu, S.Y. Li, X.X. Chu, A.Y. Hao, RSC Advances 4, (2014), 4237242375 . 
[46] P. Huang, D.L. Wang, Y. Su, W. Huang, Y.F. Zhou, D.X. Cui, X.Y. Zhu, D.Y. Yan, J. Am. Chem. Soc. 136, (2014), 11748-11756.

[47] T. Zhang, P. Huang, L. Shi, Y. Su, L. Zhou, X. Zhu, D. Yan, Mol. Pharm. 12, (2015), 2328-2336.

[48] H. Su, J.M. Koo, H. Cui, J. Control. Release, doi:10.1016/j.jconrel.2015.1009.1056.

[49] R. Duncan, Nat. Rev. Cancer. 6, (2006), 688-701.

[50] E. Gianasi, M. Wasil, E.G. Evagorou, A. Keddle, G. Wilson, R. Duncan, Eur. J. Cancer 35, (1999), 994-1002.

[51] T. Minko, P. Kopečková, J. Kopeček, Pharm. Res. 16, (1999), 986-996.

[52] J. Kopeček, P. Kopečková, T. Minko, Z.-R. Lu, Eur. J. Pharm. Biopharm. 50, (2000), 61-81.

[53] R. Bhatt, P. de Vries, J. Tulinsky, G. Bellamy, B. Baker, J.W. Singer, P. Klein, J. Med. Chem. 46, (2003), 190-193.

[54] J.W. Singer, J. Control. Release 109, (2005), 120-126.

[55] M. Yokoyama, M. Miyauchi, N. Yamada, T. Okano, Y. Sakurai, K. Kataoka, S. Inoue, Cancer Res. 50, (1990), 1693-1700.

[56] M. Yokoyama, T. Okano, Y. Sakurai, K. Kataoka, J. Control. Release 32, (1994), 269-277.

[57] M. Yokoyama, G.S. Kwon, T. Okano, Y. Sakurai, H. Ekimoto, K. Okamoto, H. Mashiba, T. Seto, K. Kataoka, Drug Deliv. 1, (1993), 11-19.

[58] T. Nakanishi, S. Fukushima, K. Okamoto, M. Suzuki, Y. Matsumura, M. Yokoyama, T. Okano, Y. Sakurai, K. Kataoka, J. Control. Release 74, (2001), 295-302.

[59] Y. Matsumura, T. Hamaguchi, T. Ura, K. Muro, Y. Yamada, Y. Shimada, K. Shirao, T. Okusaka, H. Ueno, M. Ikeda, N. Watanabe, Br J Cancer 91, (2004), 1775-1781.

[60] A. Gabizon, R. Catane, B. Uziely, B. Kaufman, T. Safra, R. Cohen, F. Martin, A. Huang, Y. Barenholz, Cancer Res. 54, (1994), 987-992.

[61] Y. Tsukioka, Y. Matsumura, T. Hamaguchi, H. Koike, F. Moriyasu, T. Kakizoe, Jpn. J. Cancer Res. 93, (2002), 1145-1153.

[62] T. Hamaguchi, Y. Matsumura, M. Suzuki, K. Shimizu, R. Goda, I. Nakamura, I. Nakatomi, M. Yokoyama, K. Kataoka, T. Kakizoe, Br J Cancer 92, (2005), 1240-1246.

[63] T. Hamaguchi, T. Doi, T. Eguchi-Nakajima, K. Kato, Y. Yamada, Y. Shimada, N. Fuse, A. Ohtsu, S.-i. Matsumoto, M. Takanashi, Y. Matsumura, Clinical Cancer Research 16, (2010), 5058-5066.

[64] Z.G. Xu, K.L. Zhang, C.L. Hou, D.D. Wang, X.Y. Liu, X.J. Guan, X.Y. Zhang, H.X. Zhang, J Mater Chem B 2, (2014), 3433-3437.

[65] Z.G. Xu, D.D. Wang, S. Xu, X.Y. Liu, X.Y. Zhang, H.X. Zhang, Chem-Asian J 9, (2014), 199-205.

[66] N. Nishiyama, S. Okazaki, H. Cabral, M. Miyamoto, Y. Kato, Y. Sugiyama, K. Nishio, Y. Matsumura, K. Kataoka, Cancer Res. 63, (2003), 8977-8983.

[67] M. Yokoyama, T. Okano, Y. Sakurai, S. Suwa, K. Kataoka, J. Control. Release 39, (1996), 351356.

[68] N. Nishiyama, K. Kataoka, J. Control. Release 74, (2001), 83-94.

[69] N. Nishiyama, Y. Kato, Y. Sugiyama, K. Kataoka, Pharm. Res. 18, (2001), 1035-1041.

[70] N. Nishiyama, M. Yokoyama, T. Aoyagi, T. Okano, Y. Sakurai, K. Kataoka, Langmuir 15, (1999), 377-383.

[71] H. Uchino, Y. Matsumura, T. Negishi, F. Koizumi, T. Hayashi, T. Honda, N. Nishiyama, K. Kataoka, S. Naito, T. Kakizoe, Br. J. Cancer 93, (2005), 678-687.

[72] K. Endo, T. Ueno, S. Kondo, N. Wakisaka, S. Murono, M. Ito, K. Kataoka, Y. Kato, T. Yoshizaki, Cancer Sci. 104, (2013), 369-374.

[73] R. Plummer, R.H. Wilson, H. Calvert, A.V. Boddy, M. Griffin, J. Sludden, M.J. Tilby, M. Eatock, D.G. Pearson, C.J. Ottley, Y. Matsumura, K. Kataoka, T. Nishiya, Br. J. Cancer 104, (2011), 593598.

[74] Y. Matsumura, H. Maeda, Cancer Res. 46, (1986), 6387-6392.

[75] V. Torchilin, Adv. Drug Del. Rev. 63, (2011), 131-135.

[76] X. Hu, J. Tian, T. Liu, G. Zhang, S. Liu, Macromolecules 46, (2013), 6243-6256.

[77] D.Y. Furgeson, M.R. Dreher, A. Chilkoti, J. Control. Release 110, (2006), 362-369. 
[78] J.A. MacKay, M. Chen, J.R. McDaniel, W. Liu, A.J. Simnick, A. Chilkoti, Nat. Mater. 8, (2009), 993-999.

[79] J.R. McDaniel, J. Bhattacharyya, K.B. Vargo, W. Hassouneh, D.A. Hammer, A. Chilkoti, Angew. Chem. Int. Ed. 52, (2013), 1683-1687.

[80] W.R. Gray, L.B. Sandberg, J.A. Foster, Nature 246, (1973), 461-466.

[81] D.E. Meyer, G.A. Kong, M.W. Dewhirst, M.R. Zalutsky, A. Chilkoti, Cancer Res. 61, (2001), 1548-1554.

[82] D. Raucher, A. Chilkoti, Cancer Res. 61, (2001), 7163-7170.

[83] E.M. Mastria, M. Chen, J.R. McDaniel, X. Li, J. Hyun, M.W. Dewhirst, A. Chilkoti, J. Control. Release 208, (2015), 52-58.

[84] J.R. McDaniel, S.R. Macewan, M. Dewhirst, A. Chilkoti, J. Control. Release 159, (2012), 362-367.

[85] J.R. McDaniel, S.R. MacEwan, X. Li, D.C. Radford, C.D. Landon, M. Dewhirst, A. Chilkoti, Nano Lett. 14, (2014), 2890-2895.

[86] Y. Mai, A. Eisenberg, Chem. Soc. Rev. 41, (2012), 5969-5985.

[87] X. Hu, J. Hu, J. Tian, Z. Ge, G. Zhang, K. Luo, S. Liu, J. Am. Chem. Soc. 135, (2013), 1761717629.

[88] Y.G. Jin, R. Xin, L. Tong, L.N. Du, M. Li, Mol. Pharm. 8, (2011), 867-876.

[89] L.N. Du, L.L. Wu, Y.G. Jin, J.W. Jia, M. Li, Y. Wang, Intl. J. Pharm. 472, (2014), 1-9.

[90] Y. Jin, Y. Qiao, M. Li, P. Ai, X. Hou, Colloid Surface B 42, (2005), 45-51.

[91] P. Couvreur, B. Stella, L.H. Reddy, H. Hillaireau, C. Dubernet, D. Desmaële, S. Lepêtre-Mouelhi, F. Rocco, N. Dereuddre-Bosquet, P. Clayette, V. Rosilio, V. Marsaud, J.-M. Renoir, L. Cattel, Nano Lett. 6, (2006), 2544-2548.

[92] F. Dosio, L.H. Reddy, A. Ferrero, B. Stella, L. Cattel, P. Couvreur, Bioconjugate Chem. 21, (2010), 1349-1361.

[93] Y.G. Jin, Y.J. Lian, L.N. Du, S.M. Wang, C. Su, C.S. Gao, Intl. J. Pharm. 430, (2012), 276-281.

[94] M. Li, S. Qi, Y.G. Jin, J.X. Dong, Intl. J. Pharm. 478, (2015), 124-130.

[95] N. Sémiramoth, C.D. Meo, F. Zouhiri, F. Saïd-Hassane, S. Valetti, R. Gorges, V. Nicolas, J.H. Poupaert, S. Chollet-Martin, D. Desmaële, R. Gref, P. Couvreur, ACS Nano 6, (2012), 3820-3831.

[96] Z. Cheikh-Ali, J. Caron, S. Cojean, C. Bories, P. Couvreur, P.M. Loiseau, D. Desmaële, E. Poupon, P. Champy, ChemMedChem 10, (2015), 411-418.

[97] Y.Q. Shen, E.L. Jin, B. Zhang, C.J. Murphy, M.H. Sui, J. Zhao, J.Q. Wang, J.B. Tang, M.H. Fan, E. Van Kirk, W.J. Murdoch, J. Am. Chem. Soc. 132, (2010), 4259-4265.

[98] H.Q. Dong, C.Y. Dong, Y. Feng, T.B. Ren, Z.H. Zhang, L. Li, Y.Y. Li, MedChemComm 3, (2012), 1555-1561.

[99] Y. Bao, Y. Guo, X. Zhuang, D. Li, B. Cheng, S. Tan, Z. Zhang, Mol. Pharm. 11, (2014), 31963209.

[100] Y. Barenholz, J. Control. Release 160, (2012), 117-134.

[101] A.G. Cheetham, P.C. Zhang, Y.A. Lin, L.L. Lock, H.G. Cui, J. Am. Chem. Soc. 135, (2013), 29072910.

[102] W.J. Goux, L. Kopplin, A.D. Nguyen, K. Leak, M. Rutkofsky, V.D. Shanmuganandam, D. Sharma, H. Inouye, D.A. Kirschner, J. Biol. Chem. 279, (2004), 26868-26875.

[103] R. Nelson, M.R. Sawaya, M. Balbirnie, A.O. Madsen, C. Riekel, R. Grothe, D. Eisenberg, Nature 435, (2005), 773-778.

[104] A.G. Cheetham, Y.-C. Ou, P. Zhang, H. Cui, Chem. Commun. 50, (2014), 6039-6042.

[105] R. Lin, A.G. Cheetham, P. Zhang, Y.-A. Lin, H. Cui, Chem. Commun. 49, (2013), 4968-4970.

[106] A.G. Cheetham, P. Zhang, Y.A. Lin, R. Lin, H. Cui, J Mater Chem B 2, (2014), 7316-7326.

[107] Z. Li, E. Kesselman, Y. Talmon, M.A. Hillmyer, T.P. Lodge, Science 306, (2004), 98-101.

[108] Z. Li, M.A. Hillmyer, T.P. Lodge, Nano Lett. 6, (2006), 1245-1249.

[109] Y.-A. Lin, Y.-C. Ou, A.G. Cheetham, H. Cui, ACS Macro Lett. 2, (2013), 1088-1094.

[110] Y.-A. Lin, A.G. Cheetham, P. Zhang, Y.-C. Ou, Y. Li, G. Liu, D. Hermida-Merino, I.W. Hamley, H. Cui, ACS Nano 8, (2014), 12690-12700. 
[111] J.D. Hartgerink, E. Beniash, S.I. Stupp, Proc. Natl. Acad. Sci. USA 99, (2002), 5133-5138.

[112] J.P. Schneider, D.J. Pochan, B. Ozbas, K. Rajagopal, L. Pakstis, J. Kretsinger, J. Am. Chem. Soc. 124, (2002), 15030-15037.

[113] L.E.R. O'Leary, J.A. Fallas, E.L. Bakota, M.K. Kang, J.D. Hartgerink, Nat. Chem. 3, (2011), 821828.

[114] S. Soukasene, D.J. Toft, T.J. Moyer, H. Lu, H.-K. Lee, S.M. Standley, V.L. Cryns, S.I. Stupp, ACS Nano 5, (2011), 9113-9121.

[115] D.J. Toft, T.J. Moyer, S.M. Standley, Y. Ruff, A. Ugolkov, S.I. Stupp, V.L. Cryns, ACS Nano 6, (2012), 7956-7965.

[116] A. Ghosh, M. Haverick, K. Stump, X. Yang, M.F. Tweedle, J.E. Goldberger, J. Am. Chem. Soc. 134, (2012), 3647-3650.

[117] S.H. Kim, J.A. Kaplan, Y. Sun, A. Shieh, H.L. Sun, C.M. Croce, M.W. Grinstaff, J.R. Parquette, Chem.-Eur. J. 21, (2015), 101-105.

[118] M. Yang, D.W. Xu, L.H. Jiang, L. Zhang, D. Dustin, R. Lund, L. Liu, H. Dong, Chem. Commun. 50, (2014), 4827-4830.

[119] R. Tian, H. Wang, R. Niu, D. Ding, J. Colloid Interf. Sci. 453, (2015), 15-20.

[120] N.L. Rosi, D.A. Giljohann, C.S. Thaxton, A.K.R. Lytton-Jean, M.S. Han, C.A. Mirkin, Science 312, (2006), 1027-1030.

[121] J.I. Cutler, E. Auyeung, C.A. Mirkin, J. Am. Chem. Soc. 134, (2012), 1376-1391.

[122] X. Tan, B.B. Li, X. Lu, F. Jia, C. Santori, P. Menon, H. Li, B. Zhang, J.J. Zhao, K. Zhang, J. Am. Chem. Soc. 137, (2015), 6112-6115.

[123] D.A. Giljohann, D.S. Seferos, P.C. Patel, J.E. Millstone, N.L. Rosi, C.A. Mirkin, Nano Lett. 7 , (2007), 3818-3821.

[124] J.I. Cutler, K. Zhang, D. Zheng, E. Auyeung, A.E. Prigodich, C.A. Mirkin, J. Am. Chem. Soc. 133, (2011), 9254-9257.

[125] P.C. Patel, D.A. Giljohann, W.L. Daniel, D. Zheng, A.E. Prigodich, C.A. Mirkin, Bioconjugate Chem. 21, (2010), 2250-2256.

[126] C.H.J. Choi, L. Hao, S.P. Narayan, E. Auyeung, C.A. Mirkin, Proc. Natl. Acad. Sci. USA 110, (2013), 7625-7630.

[127] K. Zhang, L. Hao, S.J. Hurst, C.A. Mirkin, J. Am. Chem. Soc. 134, (2012), 16488-16491.

[128] P. Zhang, A.G. Cheetham, L.L. Lock, Y. Li, H. Cui, Current Opinion in Biotechnology 34, (2015), 171-179.

[129] G.A. Silva, C. Czeisler, K.L. Niece, E. Beniash, D.A. Harrington, J.A. Kessler, S.I. Stupp, Science 303, (2004), 1352-1355.

[130] M. Zhou, A.M. Smith, A.K. Das, N.W. Hodson, R.F. Collins, R.V. Ulijn, J.E. Gough, Biomaterials 30, (2009), 2523-2530.

[131] V. Jayawarna, M. Ali, T.A. Jowitt, A.F. Miller, A. Saiani, J.E. Gough, R.V. Ulijn, Adv. Mater. 18, (2006), 611-614.

[132] J.D. Hartgerink, E. Beniash, S.I. Stupp, Science 294, (2001), 1684-1688.

[133] C.J. Newcomb, R. Bitton, Y.S. Velichko, M.L. Snead, S.I. Stupp, Small 8, (2012), 2195-2202.

[134] Z. Yang, G. Liang, M. Ma, A.S. Abbah, W.W. Lu, B. Xu, Chem. Commun., (2007), 843-845.

[135] R. Li, C. Shu, W. Wang, X. Wang, H. Li, D. Xu, W. Zhong, J. Pharm. Sci. 104, (2015), 2266-2275.

[136] A. Altunbas, D. Pochan. in Peptide-Based Materials Vol. 310 Topics in Current Chemistry (ed Timothy Deming) Ch. 206, 135-167 (Springer Berlin Heidelberg, 2012).

[137] J.S. Castro, P.A. Deymier, B. Trzaskowski, J. Bucay, Colloid Surface B 76, (2010), 199-206.

[138] J.S. Castro, L. V.Tapia, R.A. Silveyra, C.A. Martinez, P.A. Deymier. in Current Cancer Treatment - Novel Beyond Conventional Approaches (ed Prof. Oner Ozdemir) (InTech, 2011).

[139]R. Cherif-Cheikh, F. Bismuth, M.L. Torres, R. Alloza, M.T. Bosch, M. Montes, E. Fuster, J. Valles, J.A. Cordero, C. Peraire, R. Obach, A. R., Proc. Intl. Symp. Control. Release Bioact. Mater. 25, (1998), 798-799.

[140] S.K. Maji, D. Schubert, C. Rivier, S. Lee, J.E. Rivier, R. Riek, PLoS Biol. 6, (2008), e17. 
[141] B. Xing, C.-W. Yu, K.-H. Chow, P.-L. Ho, D. Fu, B. Xu, J. Am. Chem. Soc. 124, (2002), 1484614847.

[142] B. Xing, P.L. Ho, C.-W. Yu, K.-H. Chow, H. Gu, B. Xu, Chem. Commun., (2003), 2224-2225.

[143]Z.M. Yang, Y. Kuang, X.M. Li, N. Zhou, Y. Zhang, B. Xu, Chem. Commun. 48, (2012), 9257-9259.

[144] Y. Gao, Y. Kuang, Z.F. Guo, Z.H. Guo, I.J. Krauss, B. Xu, J. Am. Chem. Soc. 131, (2009), 1357613577.

[145] H. Liu, Y.L. Li, Z.L. Lyu, Y.B. Wan, X.H. Li, H.B. Chen, H. Chen, X.M. Li, J Mater Chem B 2, (2014), 8303-8309.

[146] G.J. Pu, C.H. Ren, D.X. Li, L. Wang, J.T. Sun, RSC Advances 4, (2014), 50145-50147.

[147] L. Mao, H. Wang, M. Tan, L. Ou, D. Kong, Z. Yang, Chem. Commun. 48, (2012), 395-397.

[148] X.M. Li, J.Y. Li, Y.A. Gao, Y. Kuang, J.F. Shi, B. Xu, J. Am. Chem. Soc. 132, (2010), 1770717709.

[149] J.Y. Li, Y. Kuang, Y. Gao, X.W. Du, J.F. Shi, B. Xu, J. Am. Chem. Soc. 135, (2013), 542-545.

[150] X.Y. Li, Y.Q. Wang, C.B. Yang, S. Shi, L. Jin, Z.C. Luo, J. Yu, Z.L. Zhang, Z.M. Yang, H. Chen, Nanoscale 6, (2014), 14488-14494.

[151] J. Li, Y. Kuang, J. Shi, Y. Gao, J. Zhou, B. Xu, Beilstein J. Org. Chem. 9, (2013), 908-917.

[152] J.Y. Li, X.M. Li, Y. Kuang, Y. Gao, X.W. Du, J.F. Shi, B. Xu, Adv. Healthcare Mater. 2, (2013), 1586-1590.

[153] J.D. Carmichael, Patient Prefer. Adherence 6, (2012), 73-82.

[154] M.E. Caplin, M. Pavel, J.B. Ćwikła, A.T. Phan, M. Raderer, E. Sedláčková, G. Cadiot, E.M. Wolin, J. Capdevila, L. Wall, G. Rindi, A. Langley, S. Martinez, J. Blumberg, P. Ruszniewski, New Engl. J. Med. 371, (2014), 224-233.

[155] C. Valéry, M. Paternostre, B. Robert, T. Gulik-Krzywicki, T. Narayanan, J.-C. Dedieu, G. Keller, M.-L. Torres, R. Cherif-Cheikh, P. Calvo, F. Artzner, Proc. Natl. Acad. Sci. USA 100, (2003), 10258-10262.

[156] A. Mahler, M. Reches, M. Rechter, S. Cohen, E. Gazit, Adv. Mater. 18, (2006), 1365-1370.

[157] E. Gazit, FASEB J. 16, (2002), 77-83.

[158] C.H. Görbitz, Chem.-Eur. J. 7, (2001), 5153-5159.

[159] M. Reches, E. Gazit, Science 300, (2003), 625-627.

[160] C.B. Yang, Z.Y. Wang, C.W. Ou, M.S. Chen, L. Wang, Z.M. Yang, Chem. Commun. 50, (2014), 9413-9415.

[161] C.H. Ren, C. Xu, D.X. Li, H. Ren, J.H. Hao, Z.M. Yang, RSC Advances 4, (2014), 34729-34732.

[162] M.G. Kris, R.J. Gralla, R.A. Clark, L.B. Tyson, S. Groshen, Cancer 60, (1987), 2816-2822.

[163] C.B. Yang, M.J. Bian, Z.M. Yang, Biomater. Sci. 2, (2014), 651-654.

[164] K.Y. Choi, K.H. Min, J.H. Na, K. Choi, K. Kim, J.H. Park, I.C. Kwon, S.Y. Jeong, J. Mater. Chem. 19, (2009), 4102-4107.

[165]H.M. Wang, J. Wei, C.B. Yang, H.Y. Zhao, D.X. Li, Z.N. Yin, Z.M. Yang, Biomaterials 33, (2012), 5848-5853.

[166] M.J. Webber, J.B. Matson, V.K. Tamboli, S.I. Stupp, Biomaterials 33, (2012), 6823-6832.

[167] J.B. Matson, S.I. Stupp, Chem. Commun. 47, (2011), 7962-7964.

[168] M. Conda-Sheridan, S.S. Lee, A.T. Preslar, S.I. Stupp, Chem. Commun. 50, (2014), 13757-13760.

[169] J.B. Matson, M.J. Webber, V.K. Tamboli, B. Weber, S.I. Stupp, Soft Matter 8, (2012), 6689-6692.

[170] H.M. Wang, C.H. Yang, L. Wang, D.L. Kong, Y.J. Zhang, Z.M. Yang, Chem. Commun. 47, (2011), 4439-4441.

[171] Q. Xue, H. Ren, C. Xu, G. Wang, C.H. Ren, J.H. Hao, D. Ding, Sci. Rep. 5, (2015), 8764.

[172] L. J, L. J, X. H, Z. Y, C. L, L. Q, S. N, Y. C, Int. J. Nanomedicine 9, (2014), 197-207.

[173] W. Ha, J. Yu, X.Y. Song, Z.J. Zhang, Y.Q. Liu, Y.P. Shi, J Mater Chem B 1, (2013), 5532-5538.

[174] T. André, C. Louvet, F. Maindrault-Goebel, C. Couteau, M. Mabro, J.P. Lotz, V. Gilles-Amar, M. Krulik, E. Carola, V. Izrael, A. de Gramont, Eur. J. Cancer 35, 1343-1347.

[175] H. Matsuoka, K. Yano, S. Takiguchi, A. Kono, Y. Seo, T. Saito, H. Tomoda, Anticancer Res. 15, (1995), 1447-1452. 
[176] J. Majumder, J. Deb, M.R. Das, S.S. Jana, P. Dastidar, Chem. Commun. 50, (2014), 1671-1674.

[177] X. Tan, B.B. Li, X. Lu, F. Jia, C. Santori, P. Menon, H. Li, B. Zhang, J.J. Zhao, K. Zhang, J. Am. Chem. Soc., (2015).

\section{GRAPHICAL ABSTRACT}
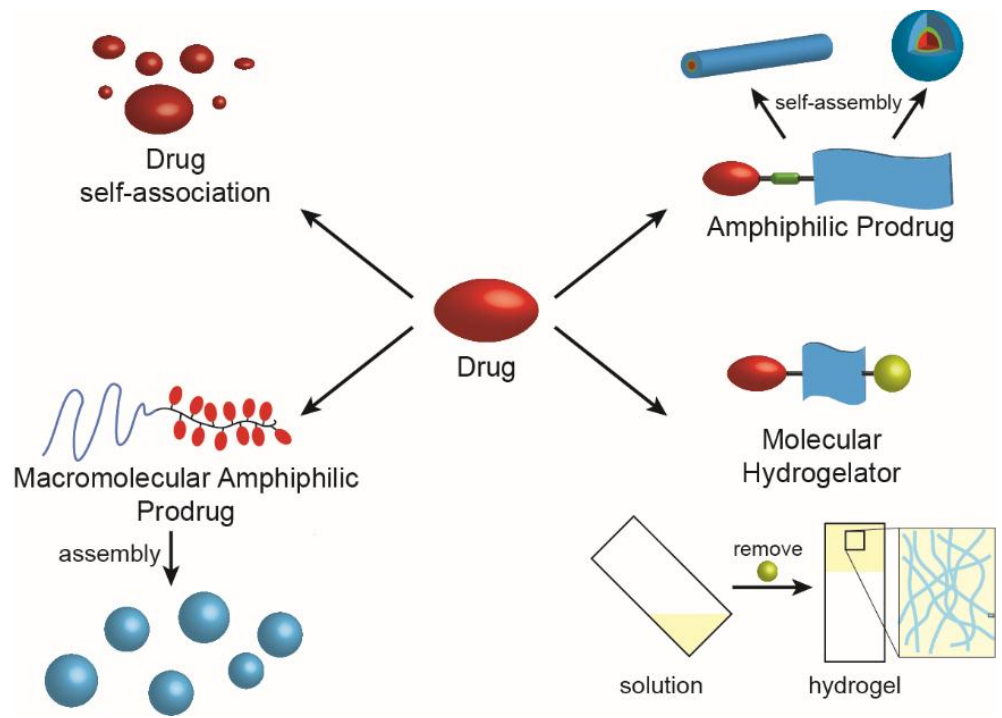


\section{FIGURES}

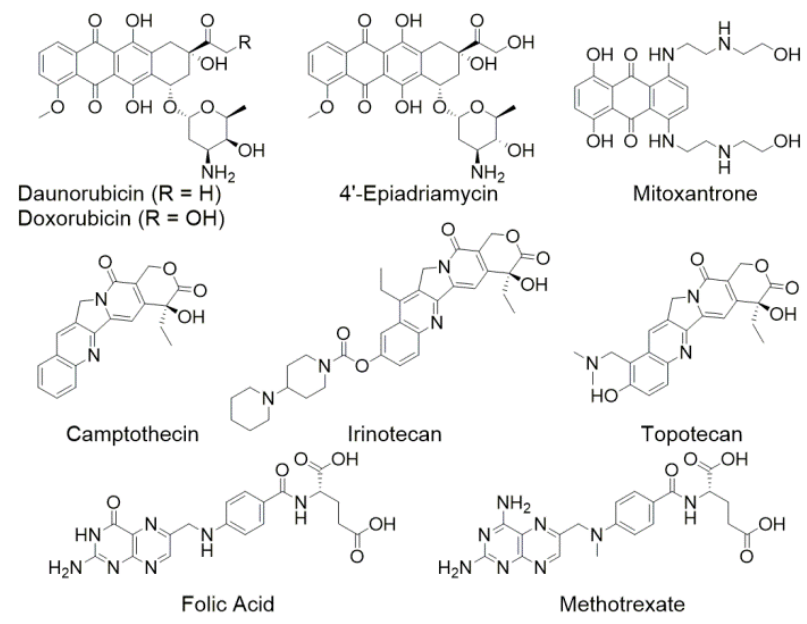

Figure 1 Molecular structures of some small molecule drugs with the potential for self-association into molecular clusters or discrete nanostructures.
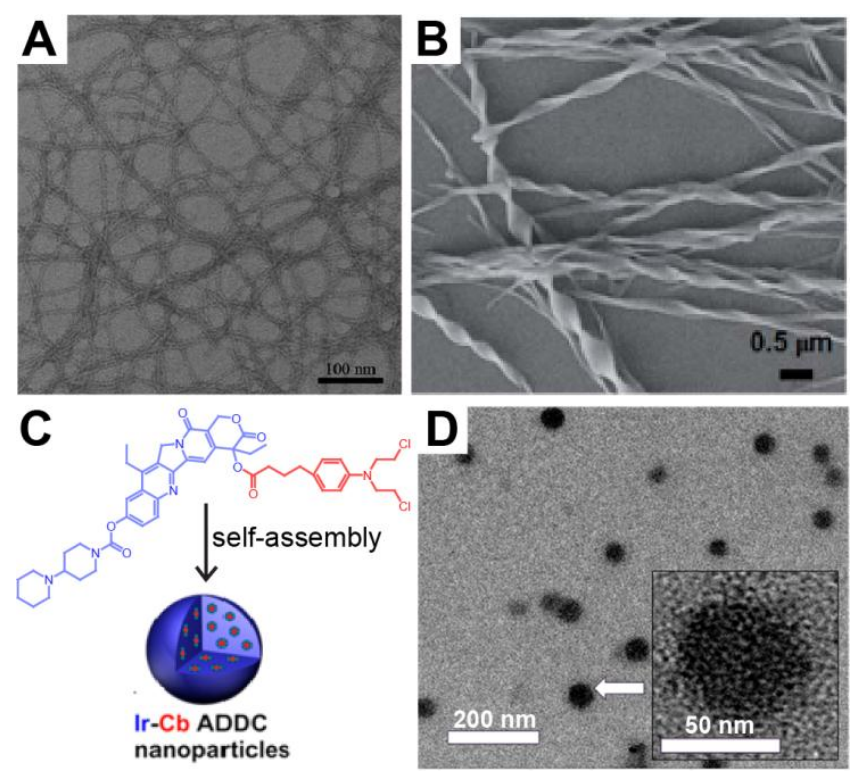

Figure 2 Representative transmission electron microscopy (TEM) images of discrete nanostructures formed by the assembly of pure drugs. (A) Cui and coworkers demonstrated how folic acid could be induced to form nanofilamentous nanostructures by the addition of water to a solution in $\mathrm{MeOH}$. Adapted with permission from ref [43], copyright 2013 Royal Society of Chemistry. (B) Hao characterized helical nanoribbons formed by camptothecin. Adapted with permission from ref [45], copyright 2014 Royal Society of Chemistry. (C-D) Yan and coworkers developed an irinotecan-chlorambacil (Ir-Cb) drug-drug conjugate (C) that could assemble into nanoparticles (D). Adapted with permission from ref [46], copyright 2014 American Chemical Society. 

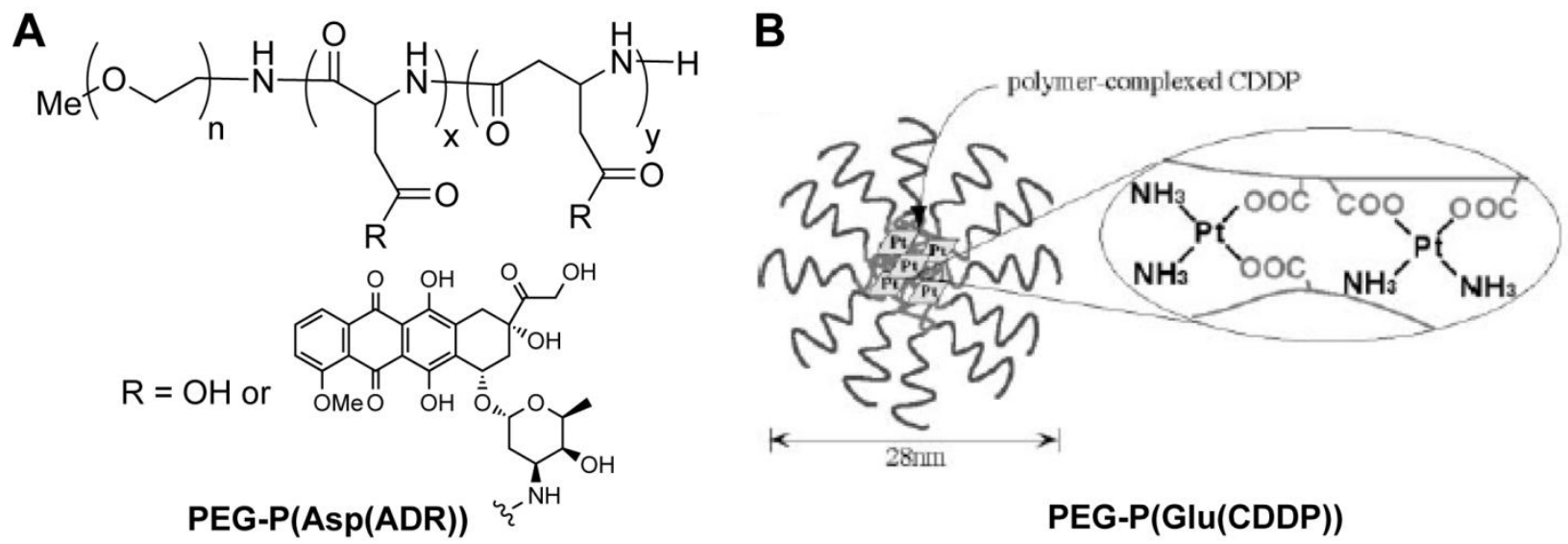

Figure 3 Macromolecular amphiphilic prodrug platform developed by Yokoyama and Kataoka. (A) PEG-P(Asp(ADR)) for the delivery of doxorubicin and (B) Pt cross-linked PEG-P(Glu) polymer for the delivery of cisplatin upon ligand exchange. Panel B was adapted with permission from ref [67], copyright 1996 Elsevier.

\section{A \\ PCL-b-P(OEGMA-CO-MAEBA-CO-FA)

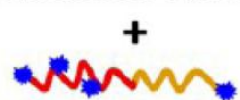 \\ Comicellization \\ P(CL-g-CPT)-b-P(OEGMA-CO-MAEBA)-CPT}

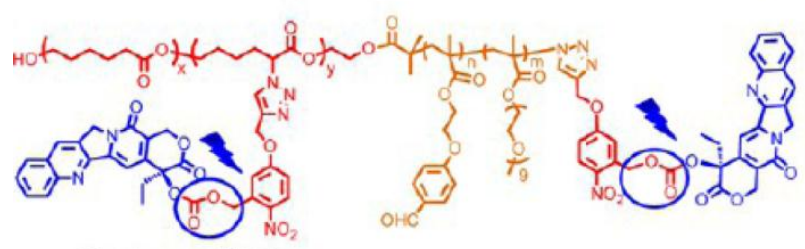

Photocaged Linkage

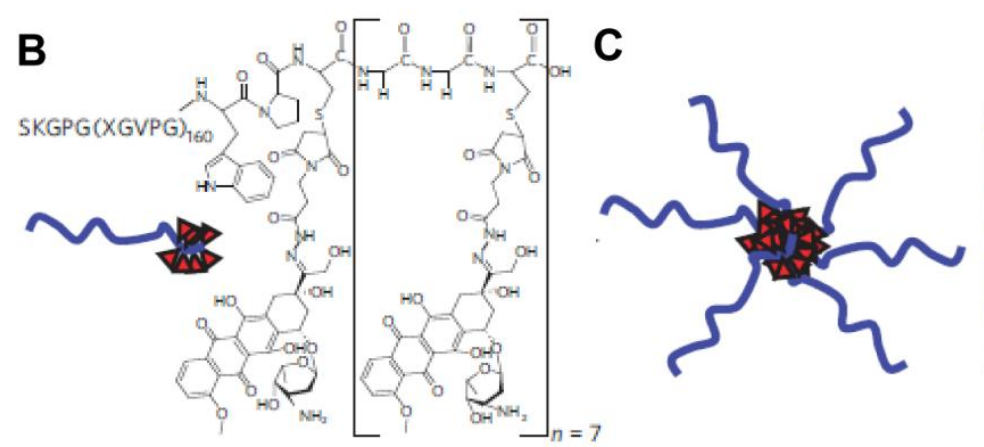

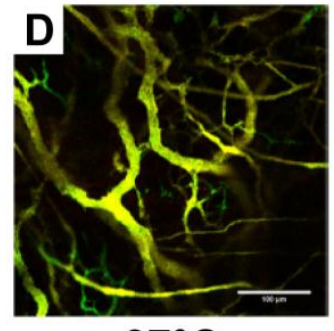

$37^{\circ} \mathrm{C}$

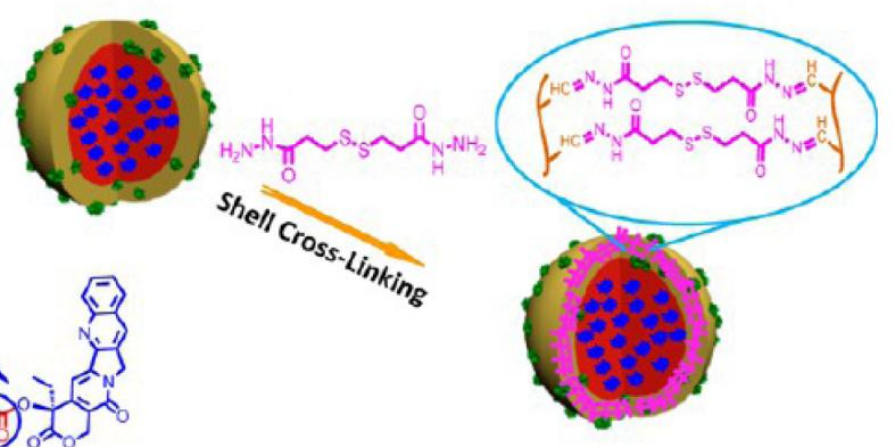

Receptor-Mediated Endocytosis Duallv Tunable Drug Release

Figure 4 Introducing greater complexity into macromolecular amphiphile prodrugs. (A) Liu's approach utilizes a photo-caged poly-CPT-containing MAP coassembled with a folic acid conjugated polymer to form a micelle that was then cross-linked with an acid- and reduction-sensitive linker. Reprinted with permission from ref [76], copyright 2013 American Chemical Society. (B) Chilkoti's ELP-based delivery platform that assembles into micelles (C) when conjugated to doxorubicin. In vivo visualization of the phase transition that occurs on heating of the tumor vasculature from $37^{\circ} \mathrm{C}$ (D) to $42^{\circ} \mathrm{C}$ (E), indicating the increased accumulation of doxorubicin within the vasculature after thermal cycling. Panels B-C were adapted with permission from ref [78], copyright 2009 Nature Publishing Group; Panels D-E were adapted with permission from ref [85], copyright 2014 American Chemical Society. 


\section{A Generic Design of Drug Amphiphiles}
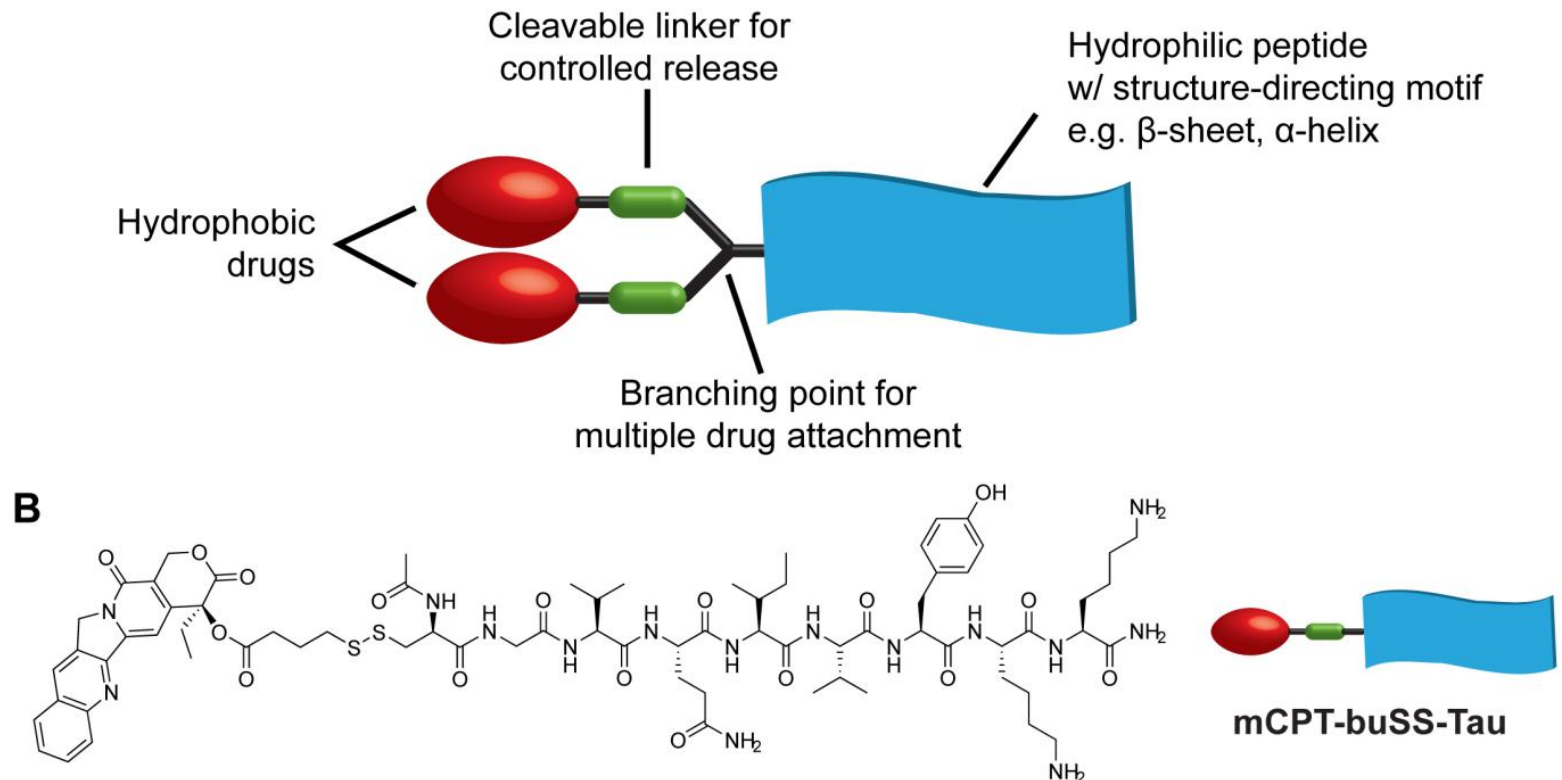

C

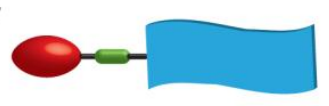

mCPT-buSS-Tau

(23\% CPT loading)

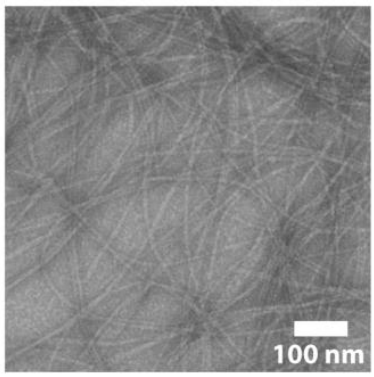

D

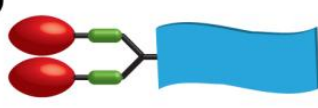

dCPT-buSS-Tau (31\% CPT loading)

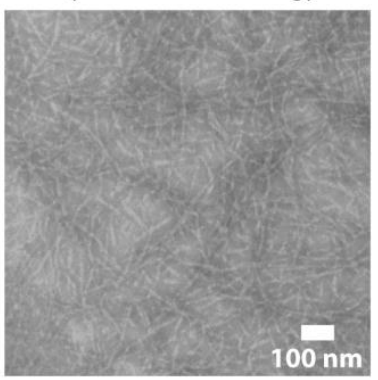

E

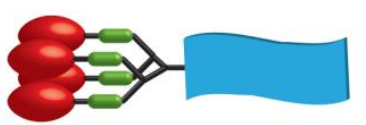

qCPT-buss-Tau

(38\% CPT loading)
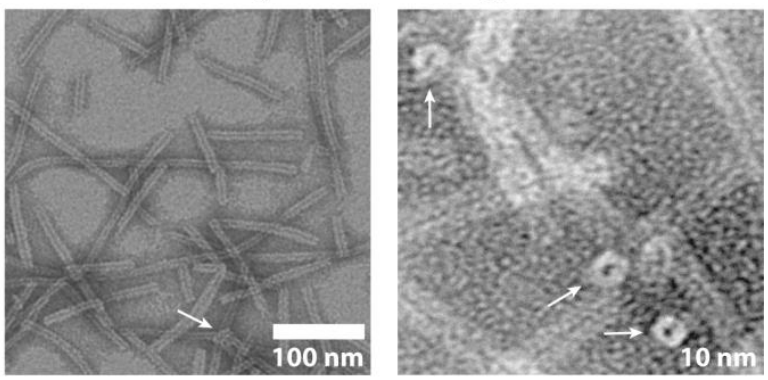

$\mathbf{F}$ single molecules
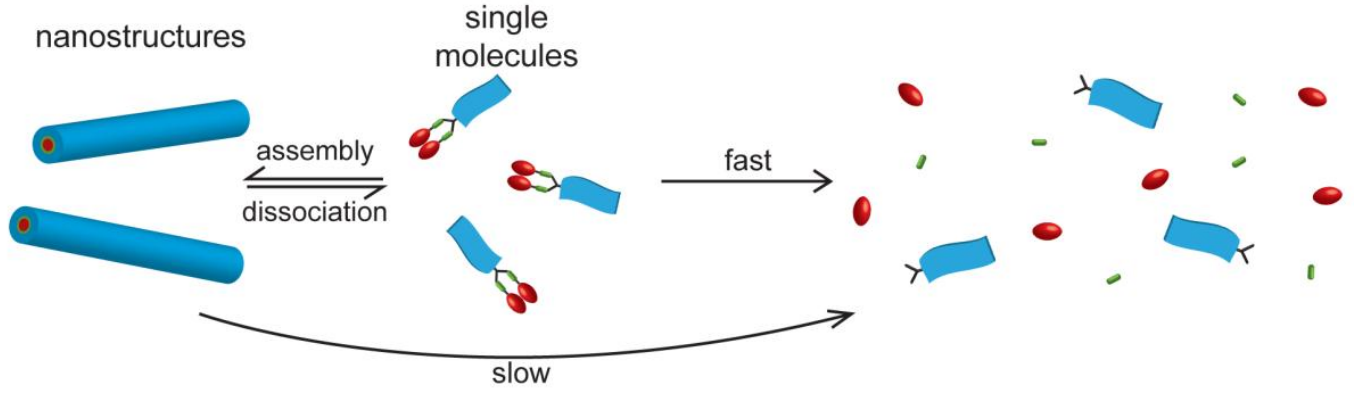

Figure 5 A self-assembling drug amphiphile platform developed by Cui and coworkers. (A) The generic design of the drug amphiphile, illustrating the essential features: a hydrophobic drug, cleavable linker and a short peptide with overall hydrophilicity and a strong preference for a particular secondary structure. A branching point allows attachment of multiple drug molecules. (B) Molecular structure of a CPT-containing drug amphiphile, mCPT-buSS-Tau, comprising a reduction-sensitive linker and a $\beta$ sheet forming Tau peptide. (C-E) Representative TEM images of the drug amphiphiles mCPT-buSS-Tau (C), dCPT-buSS-Tau (D), and qCPT-buSS-Tau (E). White arrows represent the open ends of the nanotubes formed by qCPT-buSS-Tau. (F) Schematic illustration of how assembled drug amphiphiles can act as drug reservoirs, with the assembled form protecting the linker from premature cleavage. Dissociation of the nanostructure releases the single molecule form which is vulnerable to 
breakdown, liberating the free drug. Figure elements were adapted with permission from ref [101], copyright 2013 American Chemical Society.
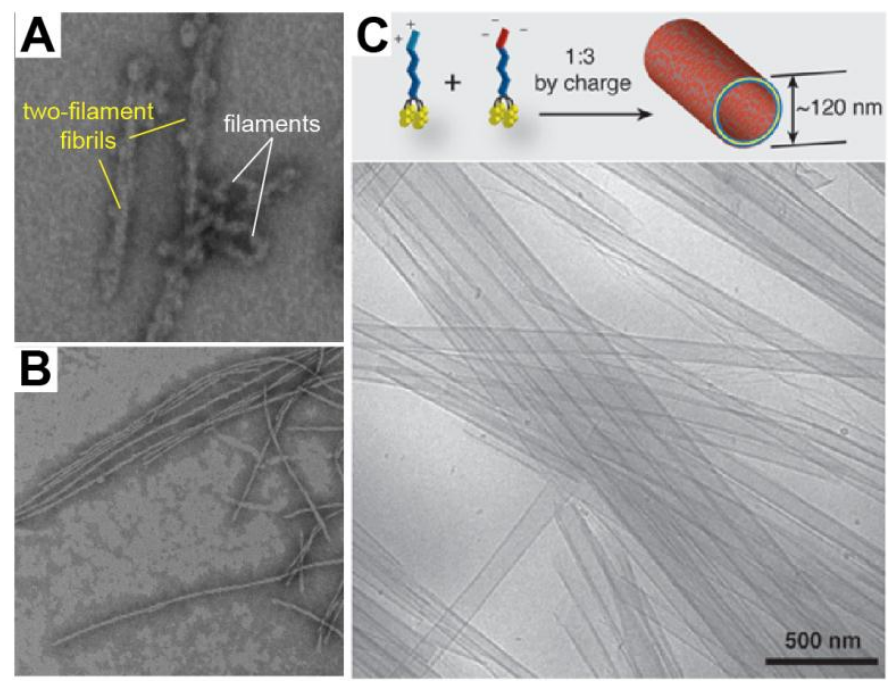

Figure 6 Construction of interesting architectures through the manipulation of the molecular packing. (A-B) Representative TEM images of a dual drug amphiphile containing both CPt and PTX, indicating the structural evolution of CPT-PTX-Sup35, which initially forms a mixture of small, flexible filaments and two-filament twisted fibrils (A), but after $24 \mathrm{~h}$ the two-filament twisted fibril nanostructure is the only structure observed (B). Adapted with permission from ref [106], copyright 2014 Royal Society of Chemistry. (C) Cryogenic TEM image of a catanionic mixture of two oppositely charged drug amphiphiles, showing the formation of form large multiwalled nanotubes. Adapted from ref [110], copyright 2014 Royal Society of Chemistry.
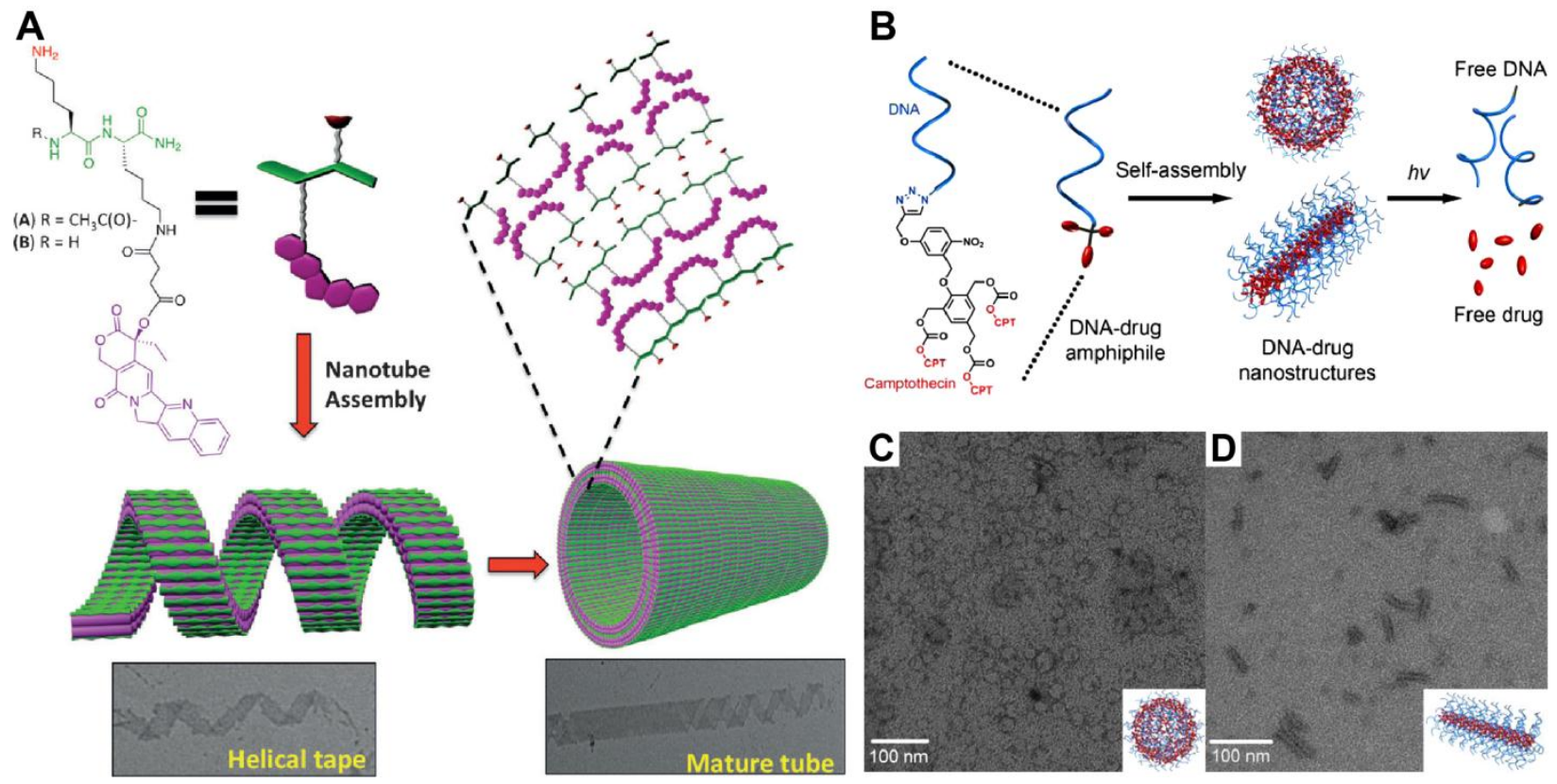

Figure 7 Alternate approaches to the creation of amphiphilic prodrugs. (A) Grinstaff and Parquette's di-lysine-based CPT conjugate that can assemble into large nanotubes via the formation and maturation of helical tape structures. Adapted with permission from ref [117], copyright 2015 Wiley-VCH. (B-D) DNA-drug amphiphile platform. Schematic illustration of the molecular structure, assembly and degradation of DNA-drug amphiphile (B). Representative TEM images of the spherical nanostructure formed by DNA $5-$ CPT in phosphate-buffered saline (PBS) (CB) and the rod-like structure formed by DNA $20-\mathrm{CPT}$ in PBS with $5 \mathrm{mM} \mathrm{MgCl}_{2}$ (D). Adapted from from ref [177], copyright 2015 American Chemical Society. 
A

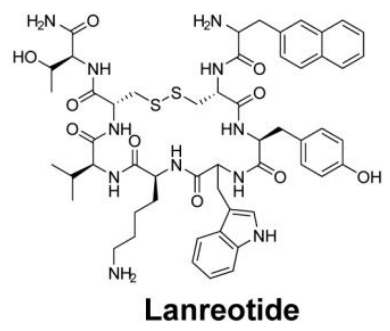

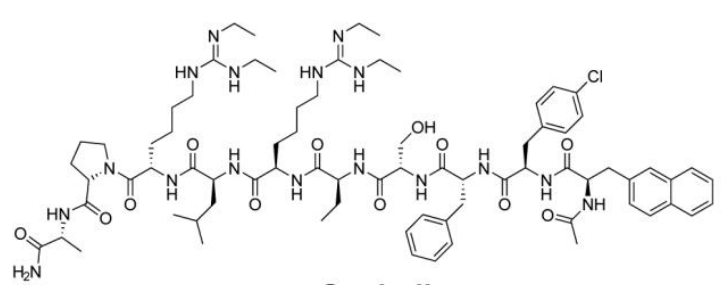

B

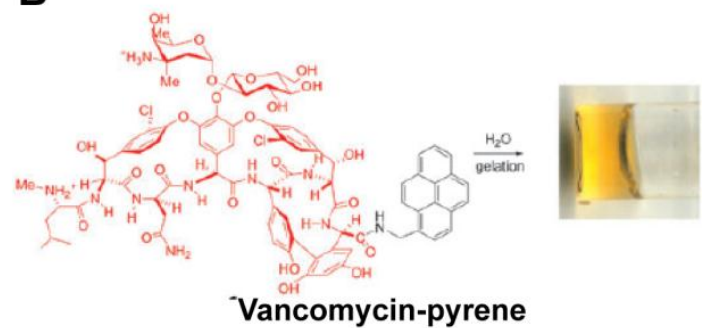

C
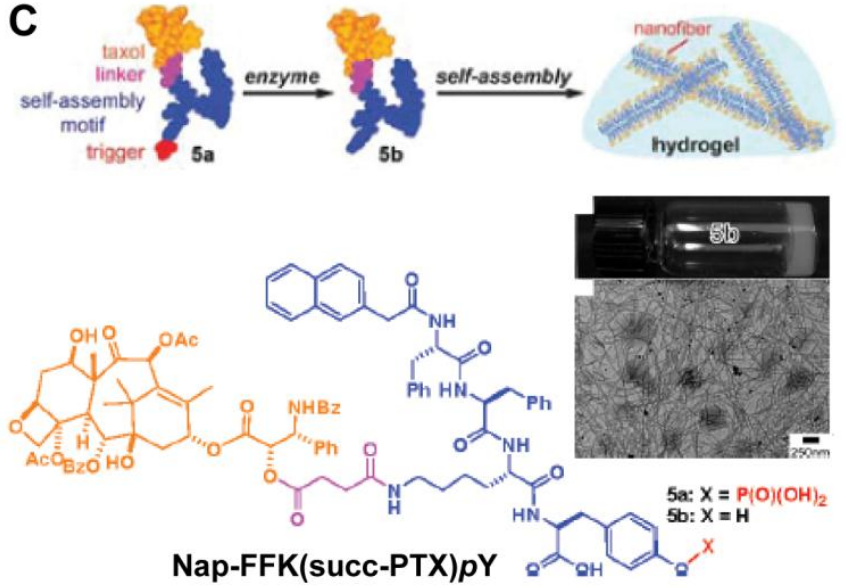

D

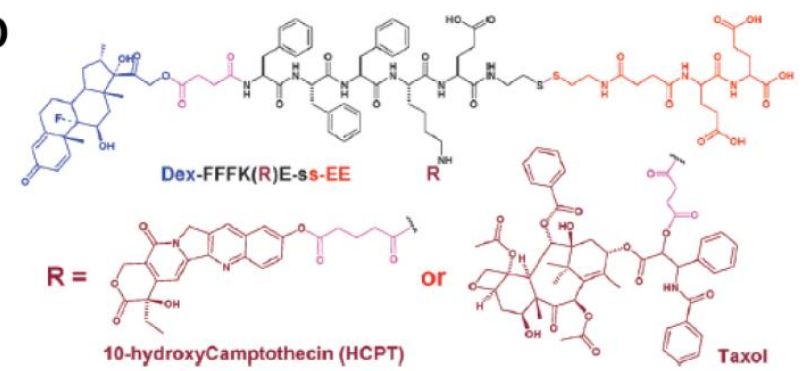

Figure 8 Examples of peptide-based molecular hydrogelators. (A-B) Peptide-based drugs that are capable of forming hydrogels either by themselves (autogels) (A) or by the addition of a hydrophobic moiety to induce gelation (B). Panel B was adapted with permission from ref [141], copyright 2002 American Chemical Society. (C) Xu's taxol-based hydrogelator, Nap-FFK(succPTX)pY, for which the phosphatase-catalysed removal of a tyrosine phosphate group decreases the solubility, triggering nanofilament formation and ultimately gelation via entanglement. Adapted with permission from ref [144], copyright 2009 American Chemical Society. (D) Yang's dual drug containing hydrogelator, in which an anticancer drug (PTX or HCPT) is paired with the anti-inflammatory/immunosuppressant, dexamethasome (DEX). Gelation is triggered by the reduction of the disulfide bond that is used to attach a hydrophilic tail. Adapted with permission from ref [147], copyright 2012 Royal Society of Chemistry. 
A
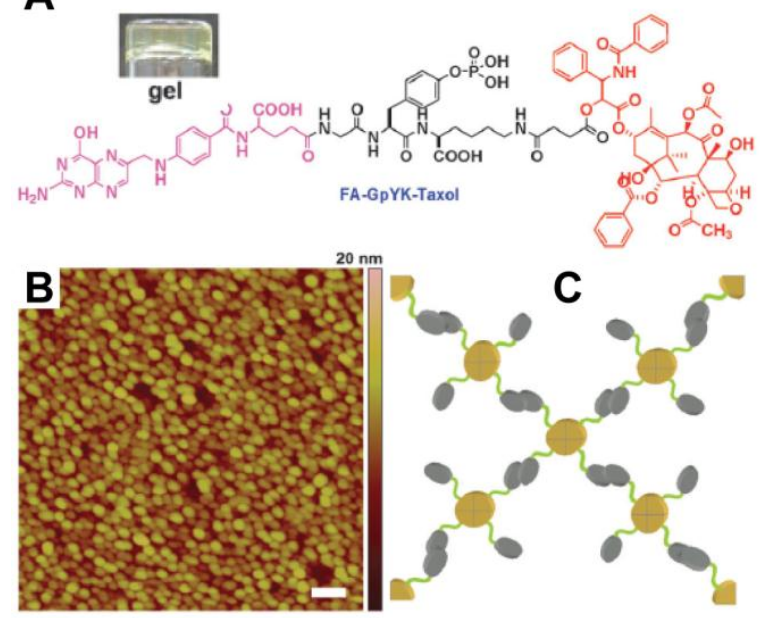

D

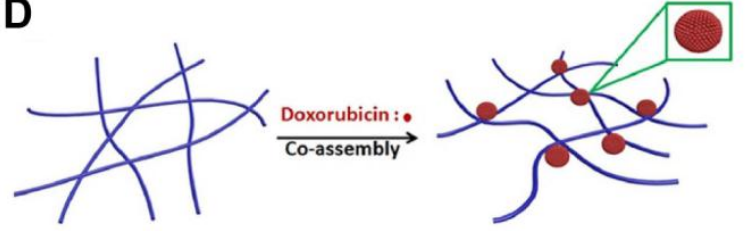

E

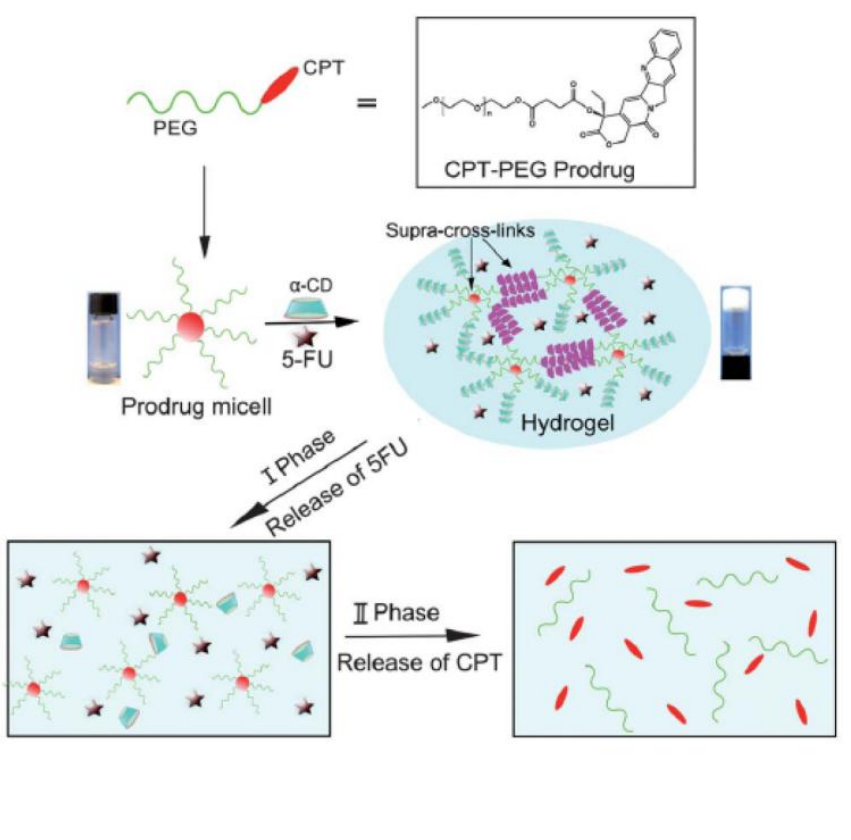

Figure 9 Alternate approaches to the creation of molecular hydrogelators. (A-C) Yang's folic acid (FA)-taxol based hydrogelator (A) that forms nanospheres rather than nanofilaments, as indicated by atomic force microscopy (AFM) (B), through Hoogsteen hydrogen bonding between FA molecules (C). Futher interactions between these nanospheres induces the formation of a weak gel. Adapted with permission from ref [170], copyright 2011 Royal Society of Chemistry. (D) Schematic illustration of Xue's platform, in which a non-gelating peptide is induced to form a hydrogel through the formation of doxorubicin nanospheres that act as cross-linkers between filaments. Reprinted with permission from ref [171], copyright 2015 Nature Publishing Group. (E) Schematic illustration of the highly supramolecular approach adopted by Shi, in which CPT-PEG micelles form polypseudorotaxane structures via threading of $\alpha$-cyclodextrin onto one or more PEG chains, thereby cross-linking and triggering gel formation. The drug 5-fluorouracil (5-FU) could also be encapsulated within the gel, with the slow dethreading of the psuedorotaxane breaking down the gel and releasing 5-FU and PEG-CPT micelles, that then underwent hydrolysis to release CPT. Reprinted from ref [173], copyright 2013 Royal Society of Chemistry. 